\title{
SYMMETRIC AND ASYMMETRIC RAMSEY PROPERTIES IN RANDOM HYPERGRAPHS
}

\author{
LUCA GUGELMANN ${ }^{1}$, RAJKO NENADOV ${ }^{2}$, YURY PERSON ${ }^{3}$, \\ NEMANJA ŠKORIĆ ${ }^{1}$, ANGELIKA STEGER ${ }^{1}$ and HENNING THOMAS ${ }^{1}$ \\ ${ }^{1}$ Institute of Theoretical Computer Science, ETH Zurich, 8092 Zurich, Switzerland; \\ email: lgugelmann@inf.ethz.ch,nskoric@inf.ethz.ch, steger@inf.ethz.ch,hthomas@inf.ethz.ch \\ ${ }^{2}$ School of Mathematical Sciences, Monash University, VIC 3800, Australia; \\ email: rajko.nenadov@monash.edu \\ ${ }^{3}$ Institute of Mathematics, Goethe-Universität, 60325 Frankfurt am Main, Germany; \\ email: person@math.uni-frankfurt.de
}

Received 18 October 2016; accepted 19 August 2017

\begin{abstract}
A celebrated result of Rödl and Ruciński states that for every graph $F$, which is not a forest of stars and paths of length 3 , and fixed number of colours $r \geqslant 2$ there exist positive constants $c, C$ such that for $p \leqslant c n^{-1 / m_{2}(F)}$ the probability that every colouring of the edges of the random graph $G(n, p)$ contains a monochromatic copy of $F$ is $o(1)$ (the ' 0 -statement'), while for $p \geqslant C n^{-1 / m_{2}(F)}$ it is $1-o(1)$ (the '1-statement'). Here $m_{2}(F)$ denotes the 2-density of $F$. On the other hand, the case where $F$ is a forest of stars has a coarse threshold which is determined by the appearance of a certain small subgraph in $G(n, p)$. Recently, the natural extension of the 1-statement of this theorem to $k$ uniform hypergraphs was proved by Conlon and Gowers and, independently, by Friedgut, Rödl and Schacht. In particular, they showed an upper bound of order $n^{-1 / m_{k}(F)}$ for the 1-statement, where $m_{k}(F)$ denotes the $k$-density of $F$. Similarly as in the graph case, it is known that the threshold for star-like hypergraphs is given by the appearance of small subgraphs. In this paper we show that another type of threshold exists if $k \geqslant 4$ : there are $k$-uniform hypergraphs for which the threshold is determined by the asymmetric Ramsey problem in which a different hypergraph has to be avoided in each colour class. Along the way we obtain a general bound on the 1-statement for asymmetric Ramsey properties in random hypergraphs. This extends the work of Kohayakawa and Kreuter, and of Kohayakawa, Schacht and Spöhel who showed a similar result in the graph case. We prove the corresponding 0 -statement for hypergraphs satisfying certain balancedness conditions.
\end{abstract}

2010 Mathematics Subject Classification: 05C80, 05D10, 05C65

(c) The Author(s) 2017. This is an Open Access article, distributed under the terms of the Creative Commons Attribution licence (http://creativecommons.org/licenses/by/4.0/), which permits unrestricted re-use, distribution, and reproduction in any medium, provided the original work is properly cited. 


\section{Introduction}

Given graphs (or hypergraphs) $G$ and $F$, we denote by

$$
G \rightarrow(F)_{r}
$$

the property that every colouring of the edges of $G$ with $r$ colours contains a monochromatic copy of $F$. If $r=2$ we simply write $G \rightarrow(F)$. For example, Ramsey's theorem shows that for every two integers $\ell, r \geqslant 2$ there exists an integer $R(\ell, r)$ such that $K_{R(\ell, r)} \rightarrow\left(K_{\ell}\right)_{r}$. In other words, every $r$-edge-colouring of a sufficiently large complete graph contains a monochromatic complete graph with $\ell$ vertices. In this paper we are interested in the case where $G$ is a binomial random graph.

A random graph $G(n, p)$ is a graph on $n$ vertices and each possible edge is present with probability $p$, independent of all other edges. The study of Ramseytype questions in random graphs was initiated by Łuczak et al. [15] where, among other results, they established the threshold for

$$
G(n, p) \rightarrow\left(K_{3}\right) .
$$

In a subsequent series of papers, Rödl and Ruciński [20-22] fully solved the edge colouring problem (up to one corner case, noticed later by Friedgut and Krivelevich [9]). To state their results we first need the following definition, which we give in the more general form for $k$-uniform hypergraphs. For any graph or hypergraph $G$ we denote by $e(G)$ and $v(G)$ the number of its edges and vertices, respectively. Set

$$
d_{k}(G):= \begin{cases}0 & \text { if } e(G)=0, \\ 1 / k & \text { if } e(G)=1, v(G)=k, \\ \frac{e(G)-1}{v(G)-k} & \text { otherwise }\end{cases}
$$

and

$$
m_{k}(G):=\max _{H \subseteq G} d_{k}(H) .
$$

We refer to $m_{k}(G)$ as the $k$-density of $G$. If $d_{k}(G)=m_{k}(G)$ we say that $G$ is $k$-balanced, and it is strictly $k$-balanced if all strict subgraphs of $G$ have smaller $k$-density. With $\Delta(G)$ we denote the maximum vertex degree of $G$.

THEOREM $1[9,20,22]$. Let $F$ be a graph with at least one edge and $r \geqslant 2$.

(i) If $F$ is a forest of stars, then

$$
\lim _{n \rightarrow \infty} \mathbb{P}\left[G(n, p) \rightarrow(F)_{r}\right]=\left\{\begin{array}{ll}
0 & \text { if } p \ll n^{-1-1 /(r(\Delta(F)-1)+1)}, \\
1 & \text { if } p \gg n^{-1-1 /(r(\Delta(F)-1)+1)}
\end{array} .\right.
$$


(ii) If $r=2$ and $F$ is a forest of stars and at least one path with exactly 3 edges, then there exists a constant $C$ such that

$$
\lim _{n \rightarrow \infty} \mathbb{P}[G(n, p) \rightarrow(F)]= \begin{cases}0 & \text { if } p \ll n^{-1 / m_{2}(F)}=n^{-1} \\ 1 & \text { if } p \geqslant C n^{-1 / m_{2}(F)}=C n^{-1}\end{cases}
$$

(iii) In all other cases there exist constants $c=c(F, r)$ and $C=C(F, r)$ such that

$$
\lim _{n \rightarrow \infty} \mathbb{P}\left[G(n, p) \rightarrow(F)_{r}\right]= \begin{cases}0 & \text { if } p \leqslant c n^{-1 / m_{2}(F)} \\ 1 & \text { if } p \geqslant C n^{-1 / m_{2}(F)}\end{cases}
$$

We refer the reader to [18] for a short proof of part (iii) of Theorem 1. Theorem 1(iii) was further strengthened by Friedgut and Krivelevich [9] and by Friedgut et al. [10] as follows. Friedgut and Krivelevich [9] proved the existence of a sharp threshold for all forests $F$ and any number of colours where (iii) of Theorem 1 applies. Friedgut et al. [10] showed the existence of a sharp threshold in the two-colour case where $F=K_{3}$. The latter result was recently extended to a more general class of graphs by Schacht and Schulenburg [27] who built on the ideas of Friedgut et al. [8].

Note that the expected number of copies of a graph $F$ in $G(n, p)$ is of order $\Theta\left(n^{v(F)} p^{e(F)}\right)$ and the expected number of edges is $\Theta\left(n^{2} p\right)$. Thus, the above result essentially states that for a balanced graph $F$ the transition from the 0 - to the 1-statement happens around the value of $p$ for which these two quantities are roughly equal. In other words, if the expected number of copies of $F$ per edge is smaller than some small constant $c^{\prime}$ then colouring without monochromatic $F$ is possible, while if this number is bigger than a large constant $C^{\prime}$ then a monochromatic $F$ always appears. This can be explained by the following intuition: if each copy of $F$ contains an edge which does not belong to any other copy of $F$ then by colouring all such edges with red and every other edge with blue clearly gives a colouring without a monochromatic copy of $F$. If, on the other hand, each edge is contained in many copies of $F$ then these must overlap heavily and a monochromatic copy is unavoidable.

There are two exceptional cases in Theorem 1: stars and paths of length 3 (that is, paths with exactly 3 edges). In the case of a star $S_{\ell}$ with $\ell$ edges it is easy to see by the pigeonhole principle that $S_{r(\ell-1)+1} \rightarrow\left(S_{\ell}\right)_{r}$ for any $r \geqslant 2$. In other words, as soon as a star on $r(\ell-1)+1$ edges appears in $G(n, p)$ it is no longer possible to colour it with $r$ colours without a monochromatic $S_{\ell}$. The threshold for this event is asymptotically smaller than $n^{-1 / m_{2}\left(S_{\ell}\right)}$. In the case of $P_{3}$ and two colours a similar phenomenon occurs. Given any cycle $C_{\ell}$ of length $\ell \geqslant 3$ we obtain a 'sunshine graph' $S_{\ell}^{*}$ by appending one pending edge to each vertex of $C_{\ell}$. For any 
odd $\ell \geqslant 5$ it holds that $S_{\ell}^{*} \rightarrow\left(P_{3}\right)$. From standard results it follows that whenever $p=c n^{-1}$ there is a small but constant probability that $G(n, p)$ contains such a sunshine graph. Accordingly the 0 -statement in (ii) cannot be of the same type as in (iii).

While the graph case was solved completely by Rödl and Ruciński in the 90's, the hypergraph case is still open. Here we consider the random hypergraph model analogue to $G(n, p): H^{k}(n, p)$ is a hypergraph on $n$ vertices and each possible hyperedge with $k$ vertices is present with probability $p$, independently of all other hyperedges. Rödl and Ruciński [23] conjectured that the same intuition should hold as for Ramsey properties in the graph case, namely that a monochromatic copy of $F$ appears in every colouring whenever the expected number of copies of $F$ per hyperedge exceeds a large constant. They proved this for the complete 3-uniform hypergraph on 4 vertices and 2 colours and, together with Schacht, extended it in [24] to $k$-partite $k$-uniform hypergraphs. Recently Friedgut et al. [11] proved the conjectured 1-statement for all $k$ uniform hypergraphs. Similar results were obtained independently by Conlon and Gowers [5].

THEOREM 2 [5, 11]. Let $F$ be a k-uniform hypergraph with maximum degree at least 2 and let $r \geqslant 2$. There exists a constant $C>0$ such that for $p=p(n)$ satisfying $\mathrm{p} \geqslant \mathrm{Cn}^{-1 / m_{k}(F)}$ we have

$$
\lim _{n \rightarrow \infty} \mathbb{P}\left[H^{k}(n, p) \rightarrow(F)_{r}\right]=1
$$

It was shown in [17] that $n^{-1 / m_{k}(F)}$ is indeed the threshold in the case where $F$ is a complete hypergraph and some further special classes were considered in [28]. However, the complete characterization, like the one in Theorem 1, is still not known. As an evidence that such a characterization might not be as simple as in the graph case, we show that there exists another type of threshold if $k \geqslant 4$. In particular, contrary to the graph case we show that there exist hypergraphs for which the threshold is neither the conjectured $n^{-1 / m_{k}(F)}$ nor is it determined by the appearance of a small subgraph. The following theorem is our first main contribution.

THEOREM 3. For every $k \geqslant 4$ there exists a $k$-uniform hypergraph $F$ and positive constants $1<\theta<m_{k}(F)$ and $c, C>0$ such that

$$
\lim _{n \rightarrow \infty} \mathbb{P}\left[H^{k}(n, p) \rightarrow(F)\right]= \begin{cases}0 & \text { if } p \leqslant c n^{-1 / \theta} \\ 1 & \text { if } p \geqslant C n^{-1 / \theta}\end{cases}
$$



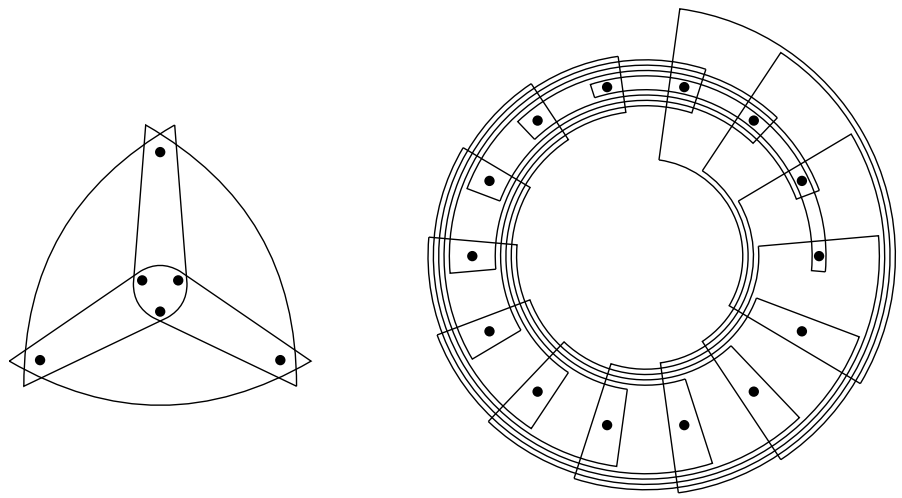

Figure 1. The hypergraph $F$ given by Theorem 3 for $k=5$.

Note that if the threshold for a hypergraph $F$ given by the previous theorem was determined by the appearance of a certain small hypergraph then, for the 0 statement to hold, we would necessarily need to have $p \ll n^{-1 / \theta}$ (we elaborate more on this in Section 2).

The $k$-uniform hypergraph $F$ in Theorem 3 is formed as a disjoint union of a hypergraph triangle and a tight cycle of certain length (see Figure 1). We formally define it in Section 2. The value of $\theta$ corresponds to the threshold for the asymmetric Ramsey property of the two hypergraphs, which we describe next. In Section 1.2, we then give an intuition behind the connection between symmetric Ramsey property for $F$ and the asymmetric one for the two hypergraphs.

1.1. Asymmetric Ramsey properties. Instead of avoiding a monochromatic copy of the same hypergraph $F$ in all colours, in the asymmetric case we want to avoid a hypergraph $F_{1}$ in red, a hypergraph $F_{2}$ in blue, and so on for all $r \geqslant 2$ colours. Similarly as before, let

$$
G \rightarrow\left(F_{1}, \ldots, F_{r}\right)
$$

denote the property that every colouring of the edges of $G$ with $r$ colours contains at least one monochromatic copy of $F_{i}$ in its respective colour (for some $1 \leqslant i \leqslant$ $r$ ). Clearly, if all $F_{i}$ are equal this reduces to the previously discussed (symmetric) case.

In the context of random graphs, the asymmetric Ramsey property was first studied by Kohayakawa and Kreuter [13] where they determined the threshold for the case where each $F_{i}$ is a cycle. They also conjectured that in the general case the 
threshold is determined by the function below. Here we state the extension for $k$ uniform hypergraphs, while the original conjecture concerns only the case $k=2$.

DEFINITION 4. Let $F_{1}, F_{2}$ be two $k$-uniform hypergraphs with at least one edge and such that $m_{k}\left(F_{1}\right) \geqslant m_{k}\left(F_{2}\right)$. The asymmetric $k$-density is defined as follows,

$$
m_{k}\left(F_{1}, F_{2}\right)=\max \left\{\frac{e\left(F_{1}^{\prime}\right)}{v\left(F_{1}^{\prime}\right)-k+1 / m_{k}\left(F_{2}\right)}: F_{1}^{\prime} \subseteq F_{1}, e\left(F_{1}^{\prime}\right) \geqslant 1\right\} .
$$

Note that if $m_{k}\left(F_{1}\right)=m_{k}\left(F_{2}\right)$ then $m_{k}\left(F_{1}, F_{2}\right)=m_{k}\left(F_{1}\right)$ and otherwise $m_{k}\left(F_{2}\right)<m_{k}\left(F_{1}, F_{2}\right)<m_{k}\left(F_{1}\right)$. We say that $F_{1}$ is strictly balanced with respect to $m_{k}\left(\cdot, F_{2}\right)$ if no proper subgraph $F_{1}^{\prime} \subsetneq F_{1}$ with at least one edge maximizes (2).

The intuition behind the conjectured value $n^{-1 / m_{k}\left(F_{1}, F_{2}\right)}$ for the asymmetric Ramsey property is easiest explained in the case $r=3$ and $F_{2}=F_{3}$. In other words, we have three colours and we aim to avoid a copy of $F_{1}$ in colour 1 and a copy of $F_{2}$ in colours 2 and 3 . First, observe that we can assign the colour 1 to every edge which does not belong to a copy of $F_{1}$. Since $m_{k}\left(F_{1}, F_{2}\right)<m_{k}\left(F_{1}\right)$, for $p=\Theta\left(n^{-1 / m_{k}\left(F_{1}, F_{2}\right)}\right)$ we do not expect the copies of $F_{1}$ to overlap too much. Therefore, the number of edges which are left is of the same order as the number of copies of $F_{1}$, which is asymptotically $n^{v\left(F_{1}\right)} p^{e\left(F_{1}\right)}$. Assuming that these edges are randomly distributed (which is not entirely correct, but it gives a good intuition) this gives us a random hypergraph $H^{\prime}$ with edge probability $p^{*}=n^{v\left(F_{1}\right)-k} p^{e\left(F_{1}\right)}$. Next, we use colours 2 and 3 for the hyperedges in $H^{\prime}$. Now the same argument as in the symmetric case applies: if the copies of $F_{2}$ are not overlapping heavily in $H^{\prime}$ then it should be possible to assign two colours to the edges of $H^{\prime}$ such that there is no monochromatic copy of $F_{2}$, and otherwise this is unavoidable. The reasoning as before shows that we expect this transition to happen around $p^{*}=n^{-1 / m_{k}\left(F_{2}\right)}$. Putting all together, we obtain the value of $p$ given by the conjecture.

In turns out that, in order to avoid a monochromatic $F_{i}$, if $p<c n^{-1 / m_{k}\left(F_{1}, F_{2}\right)}$ the third colour is actually not needed. That is, we can assign colours 1 and 2 to $H^{\prime}$ such that both monochromatic $F_{1}$ and $F_{2}$ are avoided. This is the reason why the conjectured threshold is determined only by the two graphs with largest $k$-density. Progress towards proving the conjecture in the graph case was made by Marciniszyn et al. [16], where they confirmed it for complete graphs. They also observed that the approach of Kohayakawa and Kreuter can be used to deduce the 1-statement for all graphs $F_{1}$ and $F_{2}$ which satisfy certain mild conditions, provided that the KŁR-conjecture holds (the KŁR-conjecture was verified recently by several groups of authors $[2,6,26])$. On the other hand, Kohayakawa et al. [14] gave an alternative proof for the same result by using 


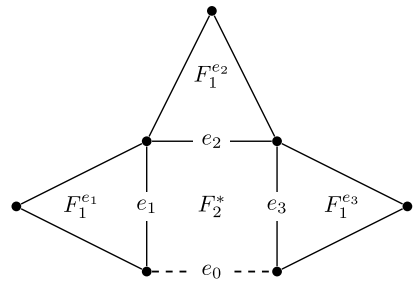

(a)

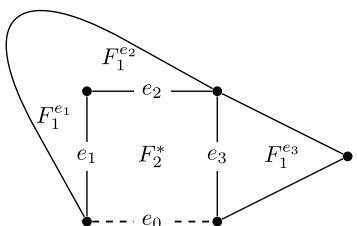

(b)

Figure 2. A generic (left) and a nongeneric (right) graph $F^{*} \in \mathcal{F}^{*}\left(K_{3}, C_{4}\right)$. Note that $F_{1}^{e_{1}}$ and $F_{1}^{e_{2}}$ are the same copy of $K_{3}$ in (b).

elementary means, with similar conditions on $F_{1}$ and $F_{2}$. Our second main contribution is an extension of these results to hypergraphs.

THEOREM 5. Let $r \geqslant 2$ and $F_{1}, \ldots, F_{r}$ be $k$-uniform hypergraphs such that $m_{k}\left(F_{1}\right) \geqslant m_{k}\left(F_{2}\right) \geqslant \cdots \geqslant m_{k}\left(F_{r}\right)$ and $F_{1}$ is strictly balanced with respect to $m_{k}\left(\cdot, F_{2}\right)$. Then there exists a constant $C>0$ such that for $p \geqslant C n^{-1 / m_{k}\left(F_{1}, F_{2}\right)}$ we have

$$
\lim _{n \rightarrow \infty} \operatorname{Pr}\left[H^{k}(n, p) \rightarrow\left(F_{1}, \ldots, F_{r}\right)\right]=1
$$

1.1.1. Sufficient criterion for the 0-statement. The corresponding 0-statement for the asymmetric Ramsey properties remains open in its full generality. As mentioned earlier, even in the case $k=2$ it is known only for some special classes of graphs, such as complete graphs and cycles. Our modest contribution towards resolving these questions is a result that reduces the problem from random graph theory to a deterministic question, at least under a certain balancedness condition. To state it we first need a couple of definitions.

Definition 6. Given $k$-uniform hypergraphs $F_{1}$ and $F_{2}$, let $\mathcal{F}^{*}\left(F_{1}, F_{2}\right)$ be the family of all $k$-uniform hypergraphs $F^{*}$ with the following property,

$$
\mathcal{F}^{*}\left(F_{1}, F_{2}\right):=\left\{\begin{array}{l}
F^{*} \text { contains a copy } F_{2}^{*} \text { of } F_{2} \text { and } \\
\text { for each } e \in E\left(F_{2}^{*}\right)-e_{0}\left(\text { for some } e_{0} \in E\left(F_{2}^{*}\right)\right) \\
\text { there exists a copy } F_{1}^{e} \text { of } F_{1}, \text { containing } e, \text { such that } \\
E\left(F^{*}\right)=E\left(F_{2}^{*}\right) \cup \bigcup_{e \in E\left(F_{2}^{*}\right)-e_{0}} E\left(F_{1}^{e}\right)
\end{array}\right\} .
$$

Note that hypergraphs $F_{1}^{e}$ need not be disjoint (in fact they are not even required to be distinct; see Figure 2b). 
Informally, every graph in the family $\mathcal{F}^{*}\left(F_{1}, F_{2}\right)$ is obtained by an amalgamation of copies of $F_{1}$ onto $F_{2}$ which cover all but at most one edge of $F_{2}$.

Given a hypergraph $F^{*} \in \mathcal{F}^{*}\left(F_{1}, F_{2}\right)$, we say that $e_{0}$ is an attachment edge of $F^{*}$. Note that there can be more than one edge which fall under the definition of an attachment edge. Moreover, we say that a member $F^{*} \in \mathcal{F}^{*}\left(F_{1}, F_{2}\right)$ is generic if the following holds: each $F_{1}^{e}$ intersects $F_{2}^{*}$ on exactly $k$ vertices (namely those which correspond to the intersecting edge $e$ ) and the remaining vertices of $F_{1}^{e}$ are disjoint from those of all other $F_{1}^{e^{\prime}}$ (see Figure 2). Observe that there could be several nonisomorphic generic copies since an attachment edge can vary, and $F_{1}$ and $F_{2}$ need not be 'symmetric'.

The main property that we require $\mathcal{F}^{*}\left(F_{1}, F_{2}\right)$ to possess resembles that of strictly $k$-balancedness.

DEFINITION 7 (Asymmetric-balanced). For given $k$-uniform hypergraphs $F_{1}$ and $F_{2}$, we say that $\mathcal{F}^{*}:=\mathcal{F}^{*}\left(F_{1}, F_{2}\right)$ is asymmetric-balanced if the following two conditions are satisfied for all $F^{*} \in \mathcal{F}^{*}$ and all $H \subsetneq F^{*}$ with $V(H) \subsetneq V\left(F^{*}\right)$ that contain an attachment edge of $F^{*}$ :

$$
\frac{e\left(F^{*}\right)-e(H)}{v\left(F^{*}\right)-v(H)} \geqslant m_{k}\left(F_{1}, F_{2}\right)
$$

(2) if

$$
\frac{e\left(F^{*}\right)-e(H)}{v\left(F^{*}\right)-v(H)}=m_{k}\left(F_{1}, F_{2}\right)
$$

then $H$ consists of exactly an attachment edge and $F^{*}$ is generic.

The next theorem shows that the function $m_{k}(\cdot, \cdot)$ indeed determines the threshold for the asymmetric Ramsey property for all $k$-uniform hypergraphs which satisfy certain conditions.

THEOREM 8. Let $F_{1}, \ldots, F_{r}$ be k-uniform hypergraphs such that $F_{2}$ has at least three edges, $m_{k}\left(F_{1}\right) \geqslant m_{k}\left(F_{2}\right) \geqslant \cdots \geqslant m_{k}\left(F_{r}\right)$ and the following holds:

(i) $F_{1}$ and $F_{2}$ are strictly balanced and $m_{k}\left(F_{2}\right) \geqslant 1$;

(ii) $F_{1}$ is strictly balanced with respect to $m_{k}\left(\cdot, F_{2}\right)$;

(iii) $\mathcal{F}^{*}\left(F_{1}, F_{2}\right)$ is asymmetric-balanced;

(iv) for every $k$-uniform hypergraph $G$ such that $m(G) \leqslant m_{k}\left(F_{1}, F_{2}\right)$ we have

$$
G \nrightarrow\left(F_{1}, F_{2}\right) .
$$


Then there exists a constant $c>0$ such that for $p \leqslant c n^{-1 / m_{k}\left(F_{1}, F_{2}\right)}$ we have

$$
\lim _{n \rightarrow \infty} \operatorname{Pr}\left[H^{k}(n, p) \rightarrow\left(F_{1}, \ldots, F_{r}\right)\right]=0 .
$$

We prove Theorem 8 in Section 4. In Section 5 we use it to derive the 0statement for pairs of hypergraphs that are used in the proof of Theorem 3.

1.2. Asymmetric meets symmetric Ramsey. Before we dive into proofs, we elaborate on the connection between the asymmetric Ramsey properties and Theorem 3. We construct the hypergraph $F$ in Theorem 3 as a disjoint union of two carefully chosen hypergraphs $F_{1}$ and $F_{2}$ with $m_{k}\left(F_{1}\right)>m_{k}\left(F_{2}\right)$ (see Figure 1). Moreover, we choose $F_{1}$ as a triangle-like hypergraph whose threshold is asymptotically below $n^{-1 / m_{k}\left(F_{1}, F_{2}\right)}$ (in particular, it is determined by the appearance of a small subgraph). The choice of $\theta:=m_{k}\left(F_{1}, F_{2}\right)$ now comes into play as follows. First observe that if $G \not \rightarrow\left(F_{1}, F_{2}\right)$ then also $G \not \rightarrow\left(F_{1} \cup F_{2}\right)$. The 0 -statement thus follows immediately from the corresponding statement for the asymmetric case. For the 1-statement we proceed as follows. Consider some 2edge-colouring of $H \sim H^{k}(n, p)$. We arbitrarily partition the vertex set of $H$ into three parts of size $n / 3$ and only consider the three induced (coloured) hypergraphs $H_{1}, H_{2}$ and $H_{3}$. By Theorem 5, if $p>\mathrm{Cn}^{-1 / \theta}$ we know that $H_{1}$ either contains a blue $F_{1}$ or a red $F_{2}$ (or both). Similarly, by reverting the colours, Theorem 5 also implies that $H_{2}$ contains a red $F_{1}$ or a blue $F_{2}$. If in this way we find a red $F_{1}$ and a red $F_{2}$ or a blue $F_{1}$ and a blue $F_{2}$, we are done, so we just have to consider the remaining two cases.

- There exists a blue $F_{1}$ and a red $F_{1}$ :

As $\theta=m_{k}\left(F_{1}, F_{2}\right)>m_{k}\left(F_{2}\right)$ it follows from Theorem 2 that there exists a monochromatic copy of $F_{2}$ in $H_{3}$, which, regardless of its colour, gives a monochromatic copy of $F$.

- There exists a red $F_{2}$ and a blue $F_{2}$ :

This is the case where the special choice of $F_{1}$ comes into play: we choose it as a hypergraph for which the (symmetric) threshold is much lower than $n^{-1 / m_{k}\left(F_{1}\right)}$, in fact, lower than $n^{-1 / \theta}$. In particular there exists $F^{\prime}$ such that $F^{\prime} \rightarrow\left(F_{1}\right)$ and $H^{k}(n, p)$ a.a.s (asymptotically almost surely, that is, with probability which tends to 1 as $n$ goes to infinity) contains $F^{\prime}$ for $p=n^{-1 / m_{k}\left(F_{1}, F_{2}\right)}$. Thus, we conclude that $H_{3}$ contains $F^{\prime}$ and, in turn, a monochromatic copy of $F_{1}$ which again implies the existence of a monochromatic $F$.

Of course, we need to show that it is possible to choose $F_{1}$ and $F_{2}$ with the desired properties. We are able to do so for $k \geqslant 4$, thus the bound in Theorem 3 . 
Another challenge is to show that for $p<c n^{-1 / \theta}$ we can colour the edges of $H^{k}(n, p)$ such that there is no red copy of $F_{1}$ and no blue copy of $F_{2}$, which implies that there is no monochromatic $F$.

The rest of the paper is organized as follows. In the next section we describe a $k$-uniform hypergraph $F=F_{1} \cup F_{2}$ and, assuming that the threshold for $H^{k}(n, p) \rightarrow\left(F_{1}, F_{2}\right)$ is $n^{-1 / m_{k}\left(F_{1}, F_{2}\right)}$, prove Theorem 3. In Section 3 we prove Theorem 5 (the 1-statement for asymmetric Ramsey properties). In the rest of the paper we prove the matching lower bound on the threshold for $H^{k}(n, p) \rightarrow\left(F_{1}\right.$, $F_{2}$ ). In Section 4 we prove Theorem 8 and then in Section 5 we verify that it can be applied with $F_{1}$ and $F_{2}$. We close with some concluding remarks in Section 6.

\section{Proof of Theorem 3}

Let $G=(V, E)$ be a graph and $W$ a set of $k-2$ additional vertices with $V \cap W=\emptyset$. We denote by $G^{+k}=\left(V^{\prime}, E^{\prime}\right)$ the $k$-uniform hypergraph obtained by adding the vertices of $W$ to each edge of $G$, that is, we set $V^{\prime}=V \cup W$ and $E^{\prime}=\{e \cup W \mid e \in E\}$. A tight $k$-cycle $C_{t}$ is a $k$-uniform hypergraph with vertex set $\left\{v_{0}, \ldots, v_{t-1}\right\}$ and the edges $\left\{v_{i}, v_{i+1}, \ldots, v_{i+k-1}\right\}$ for every $0 \leqslant i \leqslant t-1$ (the index addition is modulo $t$ ).

The following theorem implies Theorem 3.

THEOREM 9. For every $k \geqslant 4$ there exist positive constants $c, C$ such that

$$
\lim _{n \rightarrow \infty} \mathbb{P}\left[H^{k}(n, p) \rightarrow\left(K_{3}^{+k} \cup C_{t_{k}}\right)_{r}\right]= \begin{cases}0 & \text { if } p \leqslant c n^{-1 / \theta}, \\ 1 & \text { if } p \geqslant C n^{-1 / \theta}\end{cases}
$$

where

$$
\theta:=m_{k}\left(K_{3}^{+k}, C_{t_{k}}\right)
$$

and

$$
t_{4}=8, \quad t_{5}=14, \quad \text { and } t_{k}=k^{2} \quad \text { for } k \geqslant 6 .
$$

From the definition of the $k$-density $m_{k}$ we have

$$
m_{k}\left(C_{t_{k}}\right)=\frac{t_{k}-1}{t_{k}-k} \quad \text { and } \quad m_{k}\left(K_{3}^{+k}\right)=2
$$

and thus $m_{k}\left(C_{t_{k}}\right)<m_{k}\left(K_{3}^{+k}\right)$ for our choice of $t_{k}$. In addition, one easily checks that $K_{3}^{+k}$ is strictly balanced with respect to $m_{k}\left(\cdot, C_{t_{k}}\right)$. Thus,

$$
\theta=m_{k}\left(K_{3}^{+k}, C_{t_{k}}\right)=\frac{3 t_{k}-3}{2 t_{k}-k-1}<m_{k}\left(C_{t_{k}} \cup K_{3}^{+k}\right)=2 .
$$


Observe that the threshold $\theta$ from Theorem 9 does not fall into any category (i)-(iii) from Theorem 1. Indeed, the fact that $\theta<m_{k}\left(K_{3}^{+k} \cup C_{t_{k}}\right)$ excludes (ii) and (iii). We further exclude the possibility that there exists a small hypergraph whose appearance determines the threshold. To see this let $m(F)$ denote the usual density measure

$$
m(F):=\max _{\substack{H \subseteq F \\ v(H) \geqslant 1}} \frac{e(H)}{v(H)}
$$

and note that Bollobás' small subgraphs theorem [3] (for graphs) extends straightforwardly to hypergraphs. That is, for any hypergraph $F$ we have

$$
\lim _{n \rightarrow \infty} \mathbb{P}\left[H^{k}(n, p) \text { contains a copy of } F\right]=\left\{\begin{array}{ll}
0 & \text { if } p \ll n^{-1 / m(F)}, \\
1 & \text { if } p \gg n^{-1 / m(F)}
\end{array} .\right.
$$

Therefore, if the threshold in Theorem 9 is determined by the appearance of a small subgraph, as in the 0 -statement of (i) in Theorem 1, then we would necessarily have $p \ll n^{-1 / \theta}$ in order for the 0 -statement to hold, which yields a longer interval for the phase transition, contrary to what we proved.

The proof of Theorem 9 relies on Theorem 5 (which is proven in the next section) and the following lower bound on the threshold for $H^{k}(n, p) \rightarrow\left(K_{3}^{+k}\right.$, $C_{t_{k}}$ ) which we prove in Section 4.

LEMMA 10. For every $k \geqslant 4$ there exists $c>0$ such that if $p<c n^{-1 / m_{k}\left(K_{3}^{+k}, C_{t_{k}}\right)}$ then

$$
\lim _{n \rightarrow \infty} \mathbb{P}\left[H^{k}(n, p) \rightarrow\left(K_{3}^{+k}, C_{t_{k}}\right)\right]=o(1) .
$$

The choice of $t_{k}$ in Theorem 9 is based on a number of inequalities that have to be satisfied. Some of them come from the proof of Lemma 10, some others will become clear soon. In particular, the reason why we need $k \geqslant 4$ is that for small values of $k$ the calculations behave differently.

Proof of Theorem 9. Note that if $H^{k}(n, p) \nrightarrow \rightarrow\left(K_{3}^{+k}, C_{t_{k}}\right)$ then also $H^{k}(n, p) \nrightarrow \rightarrow$ $\left(K_{3}^{+k} \cup C_{t_{k}}\right)$. Therefore, Lemma 10 immediately gives the 0 -statement.

For the 1-statement we proceed as explained in Section 1.2. Recall from there that the only fact that we have to check is that

$$
\lim _{n \rightarrow \infty} \mathbb{P}\left[H^{k}(n, p) \rightarrow\left(K_{3}^{+k}\right)\right]=1 \text { for } p \geqslant C n^{-1 / \theta} .
$$

Now we use that the density of $K_{6}^{+k}$ is $m\left(K_{6}^{+k}\right)=15 /(k+4)<\theta$ (this inequality is the main reason why we require $k \geqslant 4)$. As $K_{6} \rightarrow\left(K_{3}\right)$ and thus $K_{6}^{+k} \rightarrow\left(K_{3}^{+k}\right)$ we know that $H_{3}$ contains a monochromatic copy of $K_{3}^{+k}$, which concludes the proof of the theorem. 


\section{Asymmetric Ramsey properties: 1-statement}

In this section we prove Theorem 5. We generalize an approach of Nenadov and Steger [18] based on the hypergraph containers. For further applications of this method in the context of Ramsey-type problems we refer the reader to [4, 19, 25]. The proof relies on three ingredients: Ramsey's theorem, Janson's inequality and hypergraph containers. We now state each of them.

The following theorem is a well-known quantitative strengthening of Ramsey's theorem. We include the proof for convenience of the reader.

THEOREM 11 (Folklore). Let $F_{1}, \ldots, F_{r}$ be $k$-uniform hypergraphs and $r \in \mathbb{N}$ be a constant. Then there exist constants $\alpha>0$ and $n_{0} \in \mathbb{N}$ such that for any $n \geqslant n_{0}$ and any $r$-edge-colouring of $K_{n}^{(k)}$ (the complete $k$-uniform hypergraph on $n$ vertices) there are at least $\alpha n^{v\left(F_{i}\right)}$ copies of $F_{i}$ in the colour $i$, for some $1 \leqslant i \leqslant r$.

Proof. From Ramsey's theorem we know that there exists $N=N\left(F_{1}, \ldots, F_{r}\right) \in$ $\mathbb{N}$ such that every $r$-edge-colouring of the edges of $K_{N}^{(k)}$ contains a copy of $F_{i}$ in some colour $i \in[r]$. Therefore, in any $r$-edge-colouring of $K_{n}^{(k)}$ with $n \geqslant N$, every $N$-subset of the vertices contains at least one monochromatic copy of $F_{i}$ in some colour $i$. In particular, there exists $i \in[r]$ such that in at least $\left(\begin{array}{l}n \\ N\end{array}\right) / r N$ subsets of $K_{n}^{(k)}$ we find a copy of $F_{i}$ in colour $i$. On the other hand, every copy of $F_{i}$ is contained in at most $\left(\begin{array}{c}n-v\left(F_{i}\right) \\ N-v\left(F_{i}\right)\end{array}\right) N$-subsets thus the number of different monochromatic copies of $F_{i}$ is at least

$$
\left(\begin{array}{c}
n \\
N
\end{array}\right)\left(r\left(\begin{array}{c}
n-v\left(F_{i}\right) \\
N-v\left(F_{i}\right)
\end{array}\right)\right)^{-1} \geqslant \frac{(n / N)^{N}}{r n^{N-v\left(F_{i}\right)}} \geqslant \frac{n^{v\left(F_{i}\right)}}{r N^{N}} .
$$

The theorem now follows for $\alpha=\left(r N^{N}\right)^{-1}$.

Next, we derive a bound on the expected number of copies of certain hypergraphs in $H^{k}(n, p)$.

LEMMA 12. Let $F_{1}$ and $F_{2}$ be k-uniform hypergraphs such that $m_{k}\left(F_{1}\right) \geqslant$ $m_{k}\left(F_{2}\right)>0$ and $F_{1}$ is strictly balanced with respect to $m_{k}\left(\cdot, F_{2}\right)$. Let $\mathcal{F}$ be a family of subgraphs of $K_{n}^{(k)}$ such that each member $F \in \mathcal{F}$ is a union of two distinct $F_{1}$-copies intersecting on at least one edge. For a positive constant $C$, $p=C n^{-1 / m_{k}\left(F_{1}, F_{2}\right)}$ and $H \in H^{k}(n, p)$, let $X$ be the number of hypergraphs $F \in \mathcal{F}$ contained in $H$. Then there exist a constant $\delta>0$ such that

$$
E[X] \leqslant C^{\prime} n^{k-1 / m_{k}\left(F_{2}\right)-\delta / 2} .
$$


Proof. Let $F \in \mathcal{F}$ be an arbitrary member of $\mathcal{F}$. Let $F^{\prime}, F^{\prime \prime} \subset K_{n}^{(k)}$ be two $F_{1^{-}}$ copies such that $F=F^{\prime} \cup F^{\prime \prime}$ and $e\left(F^{\prime} \cap F^{\prime \prime}\right) \geqslant 1$. Set $S:=F^{\prime} \cap F^{\prime \prime}$. From the assumption that $F_{1}$ is strictly balanced with respect to $m_{k}\left(\cdot, F_{2}\right)$ and $S$ is isomorphic to a proper subgraph of $F_{1}$ we have

$$
\frac{e(S)}{v(S)-k+1 / m_{k}\left(F_{2}\right)}<m_{k}\left(F_{1}, F_{2}\right)-\delta_{S}
$$

for some $\delta_{S}>0$. Since there are only constantly many subgraphs $S \subseteq F_{1}$, there exists a constant $\delta^{\prime}>0$ such that the previous inequality holds with $\delta_{S}=\delta^{\prime}$ for every $S \subseteq F_{1}$ with $e(S) \geqslant 1$. Using the assumption $p=C n^{-1 / m_{k}\left(F_{1}, F_{2}\right)}$, a straightforward calculation shows that the expected number of subgraphs of $H$ isomorphic to $F^{\prime} \cup F^{\prime \prime}$ is at most of order

$$
\begin{aligned}
n^{v\left(F^{\prime} \cup F^{\prime \prime}\right)} p^{e\left(F^{\prime} \cup F^{\prime \prime}\right)}=n^{2 v\left(F_{1}\right)-v(S)} p^{2 e\left(F_{1}\right)-e(S)} & =\frac{O\left(n^{2\left(k-1 / m_{k}\left(F_{2}\right)\right)}\right)}{n^{v(S)} p^{e(S)}} \\
& \stackrel{(4)}{=} O\left(n^{k-1 / m_{k}\left(F_{2}\right)-\delta / 2}\right),
\end{aligned}
$$

where $\delta>0$ depends only on $\delta^{\prime}, F_{1}$ and $F_{2}$. Since there are only constantly many ways to obtain a graph as a union of two copies of $F_{1}$, this concludes the proof.

We also need the following statement on the probability of existence of certain hypergraphs in the random hypergraph $H^{k}(n, p)$. As the proof follows almost directly from Janson's inequality, we give just a sketch of the argument.

LEMMA 13. Let $F_{1}$ and $F_{2}$ be k-uniform hypergraphs such that $m_{k}\left(F_{1}\right) \geqslant$ $m_{k}\left(F_{2}\right)>0$ and $F_{1}$ is strictly balanced with respect to $m_{k}\left(\cdot, F_{2}\right)$, and let $\varepsilon>0$ be a constant. Then there exists a constant $\beta>0$ such that the following holds for any constant $C>0$ : for $p=C n^{-1 / m_{k}\left(F_{1}, F_{2}\right)}$ and any family $\mathcal{F}$ of subgraphs of $K_{n}^{(k)}$ isomorphic to $F_{1}$ with $|\mathcal{F}| \geqslant \varepsilon n^{v\left(F_{1}\right)}$ we have

$$
\operatorname{Pr}\left[F^{\prime} \nsubseteq H^{k}(n, p) \text { for all } F^{\prime} \in \mathcal{F}\right] \leqslant e^{-\beta C n^{k-1 / m_{k}\left(F_{2}\right)}} .
$$

Proof. Let $X$ be the number of members of $\mathcal{F}$ appearing in $H^{k}(n, p)$. We bound $E[X]$ as follows,

$$
E[X]=|\mathcal{F}| p^{e\left(F_{1}\right)} \geqslant \varepsilon n^{v\left(F_{1}\right)}\left(C n^{-1 / m_{k}\left(F_{1}, F_{2}\right)}\right)^{e\left(F_{1}\right)} \geqslant \varepsilon C n^{k-1 / m_{k}\left(F_{2}\right)} .
$$

By Lemma 12 and Janson's inequality (for example, see [12, Theorem 2.14]) we have

$$
\operatorname{Pr}[X<(1-\alpha) E[X]] \leqslant e^{-\alpha^{2} E[X] / 3},
$$

for any constant $\alpha>0$. Using the estimate on $E[X]$, this implies the lemma. 
Finally, we state our main tool, a container theorem of Saxton and Thomason [26] (similar result was independently obtained by Balogh et al. [2]).

Definition 14. For a given set $S$ and $\ell \in \mathbb{N}$, let $\mathcal{T}_{\ell}(S)$ denote the family of $\ell$-tuples of subsets of $S$ (not necessarily disjoint), that is,

$$
\mathcal{T}_{\ell}(S)=\left\{T=\left(S_{1}, \ldots, S_{\ell}\right) \mid S_{i} \subseteq S \text { for } 1 \leqslant i \leqslant \ell\right\} .
$$

Given a set $S$ and an $\ell$-tuple $T=\left(S_{1}, \ldots, S_{\ell}\right) \in \mathcal{T}_{\ell}(S)$, let $|T|$ denote the size of the union of $S_{i}$ 's, that is, $|T|:=\left|\bigcup_{i \in[\ell]} S_{i}\right|$. Moreover, for a subset $S^{\prime} \subseteq S$ we write $T \subseteq S^{\prime}$ to denote that $S_{i} \subseteq S^{\prime}$ for every $i \in[\ell]$.

THEOREM 15 [26, Theorem 2.3]. Let $F$ be a $k$-uniform hypergraph with $e(F) \geqslant$ 2 and let $\varepsilon>0$ be a constant. Then there exists $\ell \in \mathbb{N}$ such that for every $n \geqslant \ell$ there exists a function $f: \mathcal{T}_{\ell}\left(E\left(K_{n}^{(k)}\right)\right) \rightarrow 2^{E\left(K_{n}^{(k)}\right)}$ with the following property: for every $F$-free $k$-uniform hypergraph $H \subseteq K_{n}^{(k)}$ there exists $T \in \mathcal{T}_{\ell}\left(E\left(K_{n}^{(k)}\right)\right)$ such that:

(a) $T \subseteq E(H) \subseteq f(T)$;

(b) the number of edges in $T$ is at most $|T| \leqslant \ell n^{k-1 / m_{k}(F)}$;

(c) the hypergraph induced by the edge set $f(T)$ contains at most $\varepsilon n^{v(F)}$ copies of $F$.

With these statements at hand, we are ready to prove Theorem 5.

Proof of Theorem 5. Let $F_{1}, \ldots, F_{r}$ be $k$-uniform hypergraphs satisfying the required conditions, that is $m_{k}\left(F_{1}\right) \geqslant m_{k}\left(F_{2}\right) \geqslant \cdots \geqslant m_{k}\left(F_{r}\right)$ and $F_{1}$ is strictly balanced with respect to $m_{k}\left(\cdot, F_{2}\right)$. Since the property

$$
H \rightarrow\left(F_{1}, \ldots, F_{r}\right)
$$

is monotone increasing, we may assume that $p=C n^{-1 / m_{k}\left(F_{1}, F_{2}\right)}$ for some constant $C>0$ which we determine later. This assumption is not necessary, but it will make calculations easier.

The overall proof strategy is as follows. If a given $k$-uniform hypergraph $H$ on $n$ vertices is not Ramsey, that is, $H \nrightarrow \rightarrow\left(F_{1}, \ldots, F_{r}\right)$, then there exists a partition $E_{1}, \ldots, E_{r} \subseteq E(H)$ such that the $k$-uniform hypergraph $G_{i}=\left(V(H), E_{i}\right)$ is $F_{i}$ free for every $1 \leqslant i \leqslant r$. We clearly think of the edges from $E_{i}$ as being coloured in colour $i$. Next, we use the container theorem, Theorem 15, to 'place' each $E_{i}$ (where $2 \leqslant i \leqslant r$ ) into some container $C_{i}$ with less than $\alpha n^{v\left(F_{i}\right)}$ copies of $F_{i}$. 
This way Theorem 11 asserts that the hypergraph $R$ on the remaining edges, that is, $R=\left([n],\left(\begin{array}{c}{[n]} \\ k\end{array}\right) \backslash \bigcup_{i=2}^{r} E_{i}\right)$ contains at least $\alpha n^{v\left(F_{1}\right)}$ copies of $F_{1}$. Assuming that $H \sim H^{k}(n, p)$, we infer that the edges from $E(H) \cap E(R)$ will be coloured in colour 1 , which will allow us to get sufficiently small probability that none of the at least $\alpha n^{v\left(F_{1}\right)}$ copies of $F_{1}$ lands in $E_{1}$ (this is an application of Janson's inequality, Lemma 13). Of course, there are some subtleties as to how we define certain probability events. In particular the options for $C_{i}$ 's and $R$ have to be considered 'beforehand' (via Theorem 15).

For $H \sim H^{k}(n, p)$ with $p$ as specified above, we expect that most copies of $F_{1}$ do not have edges in common (that is, are isolated). This will be indeed the case with high probability and the proof outline will be carried out in this case. The unlikely case will simply follow from Markov's inequality, which we first turn to.

Many non-isolated copies. Let $\mathcal{F}=\mathcal{F}\left(F_{1}, H\right)$ be the family of all subgraphs of $H$ isomorphic to $F_{1}$ and let $\mathcal{I}=\mathcal{I}\left(F_{1}, H\right) \subseteq \mathcal{F}$ be the subfamily of all isolated subgraphs, that is,

$$
\mathcal{I}=\left\{F^{\prime} \in \mathcal{F} \mid \forall F^{\prime \prime} \in \mathcal{F} \backslash\left\{F^{\prime}\right\}, E\left(F^{\prime}\right) \cap E\left(F^{\prime \prime}\right)=\emptyset\right\} .
$$

ClAIM 16. There exists a constant $\delta>0$ such that for $H \sim H^{k}(n, p)$ we a.a.s. have

$$
|\mathcal{F} \backslash \mathcal{I}| \leqslant n^{k-1 / m_{k}\left(F_{2}\right)-\delta} .
$$

Proof. By Lemma 12 we know that the expected number of hypergraphs $F \subseteq H$ which can be obtained as a union of two distinct $F_{1}$-copies intersecting on at least one edge is at most $n^{k-1 / m_{k}\left(F_{2}\right)-\delta / 2}$, for some constant $\delta>0$. Note that for each $F^{\prime} \in \mathcal{F} \backslash \mathcal{I}$ there exists $F^{\prime \prime} \in \mathcal{F}$ such that $S:=F^{\prime} \cap F^{\prime \prime}$ contains at least one edge and $F:=F^{\prime} \cup F^{\prime \prime} \subseteq H$. As there are only constantly many different copies of $F_{1}$ contained in $F=F^{\prime} \cup F^{\prime \prime}$, by previous observations we have that the expected size of $|\mathcal{F} \backslash \mathcal{I}|$ is $O\left(n^{k-1 / m_{k}\left(F_{2}\right)-\delta / 2}\right)$. From Markov's inequality we obtain that the actual number of such subgraphs is a.a.s. at most $n^{k-1 / m_{k}\left(F_{2}\right)-\delta}$.

Few nonisolated copies. Let us assume that $H$ is such that the bound in (5) holds. Next, note that for each $F^{\prime} \in \mathcal{F}$ at least one edge from $E\left(F^{\prime}\right)$ does not belong to $E_{1}$, as otherwise there exists a copy of $F_{1}$ in $G_{1}$, which is monochromatic. Moreover, we can assume that this holds for exactly one edge if $F^{\prime} \in \mathcal{I}$ : since each edge of $F^{\prime}$ belongs to exactly one copy of $F_{1}$ in $H$ (follows from the definition of $\mathcal{I}$ ), by re-colouring all but one (arbitrarily chosen) edge from $E\left(F^{\prime}\right) \backslash E_{1}$ with colour 1 we do not create a copy of $F_{1}$ in colour 1 . Since no new edge gets a colour $i \geqslant 2$, this clearly does not create a monochromatic copy of any $F_{i}$ in the corresponding colour. Next, for $i \geqslant 2$ we partition each colour class $E_{i}$ into $I_{i}$ 
('isolated' edges in $E_{i}$ ) and $L_{i}$ ('leftover' edges),

$$
I_{i}=\bigcup_{F^{\prime} \in \mathcal{I}} E\left(F^{\prime}\right) \cap E_{i} \quad \text { and } \quad L_{i}=E_{i} \backslash I_{i} .
$$

By the previous assumption (that all but exactly one edge in $F^{\prime} \in \mathcal{I}$ belong to $E_{1}$ ) we have that for each edge $e \in I:=\bigcup_{i=2}^{r} I_{i}$ there exists a unique $F_{e} \in \mathcal{I}$ with $e \in E\left(F_{e}\right)$, and $E\left(F_{e}\right) \cap E\left(F_{e^{\prime}}\right)=\emptyset$ for different edges $e, e^{\prime} \in I$. Finally, note that we can also assume that every edge in $H$ which does not belong to a copy of $F_{1}$ has colour 1 . It then follows that each edge in $L_{i}$ belongs to some $F^{\prime} \in \mathcal{F} \backslash \mathcal{I}$, and from $|\mathcal{F} \backslash \mathcal{I}| \leqslant n^{k-1 / m_{k}\left(F_{2}\right)-\delta}$ we conclude

$$
\left|L_{i}\right|=O\left(n^{k-1 / m_{k}\left(F_{2}\right)-\delta}\right) .
$$

Next, we use the container theorem to 'approximate' each of the sets $I_{i}$. Let $\alpha$ be the constant given by Theorem 11 for $F_{1}, \ldots, F_{r}$ and set $\varepsilon=\alpha / 2$. Furthermore, let $\ell_{i} \in \mathbb{N}$ and $f_{i}: \mathcal{T}_{\ell_{i}}\left(E\left(K_{n}^{(k)}\right)\right) \rightarrow 2^{E\left(K_{n}^{(k)}\right)}$ be functions obtained by applying Theorem 15 with $\varepsilon$ and $F=F_{i}$, for each $i \geqslant 2$. Since the hypergraph induced by the set of edges $I_{i}$ is $F_{i}$-free there exists an $\ell_{i}$-tuple $T^{i} \in \mathcal{T}_{\ell_{i}}\left(E\left(K_{n}^{(k)}\right)\right)$ such that

$$
T^{i} \subseteq I_{i} \subseteq f_{i}\left(T^{i}\right)
$$

Let

$$
R=E\left(K_{n}^{(k)}\right) \backslash \bigcup_{i=2}^{r}\left(f_{i}\left(T^{i}\right) \cup L_{i}\right) .
$$

Note that set $R$ is uniquely determined by $\mathbf{T}=\left(T^{2}, \ldots, T^{r}\right)$ and $\mathbf{L}=\left(L_{2}, \ldots\right.$, $\left.L_{r}\right)$, where each $L_{i} \subseteq E\left(K_{n}^{(k)}\right)$ is a subset of size $O\left(n^{k-1 / m_{k}\left(F_{2}\right)-\delta}\right)$. Therefore, we can enumerate all $R$ by going over all possible choices for $\mathbf{T}$ and $\mathbf{L}$. We refer to the set $R$ fixed by the choice of $\mathbf{T}$ and $\mathbf{L}$ as $R(\mathbf{T}, \mathbf{L})$. The following claim plays the central role in our argument.

Claim 17. Given the tuples $\mathbf{T}=\left(T^{2}, \ldots, T^{r}\right)$ and $\mathbf{L}=\left(L_{2}, \ldots, L_{r}\right)$ as described above, the hypergraph induced by the set of edges $R=R(\mathbf{T}, \mathbf{L})$ contains at least $\alpha n^{v\left(F_{1}\right)}$ copies of $F_{1}$.

Proof. From Theorem 15 we have that each $f_{i}\left(T^{i}\right)(i \geqslant 2)$ contains at most $\alpha n^{v\left(F_{i}\right)} / 2$ copies of $F_{i}$. Furthermore, the number of copies of $F_{i}$ in $f_{i}\left(T^{i}\right) \cup L_{i}$ which contain an edge from $L_{i}$ is at most $n^{v\left(F_{i}\right)-k}\left|L_{i}\right|$ and from (6) we conclude that there are $o\left(n^{v\left(F_{i}\right)}\right)$ such copies. In total, $f_{i}\left(T^{i}\right) \cup L_{i}$ contains less than $\alpha n^{v\left(F_{i}\right)}$ copies of $F_{i}$, for every $i \geqslant 2$.

Consider the auxiliary $r$-edge-colouring of $K_{n}^{(k)}$ defined as follows: an edge $e$ gets the colour 1 if $e \in R$ and otherwise it gets an arbitrary colour $i \geqslant 2$ such that $e \in f_{i}\left(T^{i}\right) \cup L_{i}$. From the previous discussion we have that each colour 
$i \geqslant 2$ contains less than $\alpha n^{v\left(F_{i}\right)}$ copies of $F_{i}$ and Theorem 11 implies that $R$ has to contain at least $\alpha n^{v\left(F_{1}\right)}$ copies of $F_{1}$.

Since $E_{i}=I_{i} \cup L_{i} \subseteq f_{i}\left(T^{i}\right) \cup L_{i}$ and each $F^{\prime} \in \mathcal{F}$ contains an edge from some $E_{i}$ with $i \geqslant 2$ we conclude that $E\left(F^{\prime}\right) \nsubseteq R$ for every $F^{\prime} \in \mathcal{F}$. That is, the hypergraph $H$ completely avoids all copies of $F_{1}$ which are contained in $R$. Recall that for $\mathbf{T}=\left(T^{2}, \ldots, T^{r}\right)$ we have $T_{j}^{i} \subseteq I_{i}$ for all $j \in\left[\ell_{i}\right]$. We define the set $T=T(\mathbf{T})$ as follows:

$$
T:=T(\mathbf{T}):=\bigcup_{i \geqslant 2} \bigcup_{j=1}^{\ell_{i}} T_{j}^{i} .
$$

Observe that for each $e \in T$ there exists a copy of $F_{1}$ in $H$ containing $e$, say $F_{e}$, such that $E\left(F_{e}\right) \cap E\left(F_{e^{\prime}}\right)=\emptyset$ for different $e, e^{\prime} \in T$ (as we have already observed this for all edges in $I \supseteq T$ ). Thus, we obtain a set of copies of $F_{1}$ in $H$ which are 'rooted' at the edges from $T$.

To summarize, if $H \nrightarrow\left(F_{1}, \ldots, F_{r}\right)$ then either $\mathcal{F} \backslash \mathcal{I}$ is bigger than $n^{k-1 / m_{k}\left(F_{2}\right)-\delta}$ or there exist $\mathbf{T}=\left(T_{2}, \ldots, T_{r}\right)$ and $\mathbf{L}=\left(L_{2}, \ldots, L_{r}\right)$ such that $H$ satisfies properties $\mathcal{P}_{1}(R)$ and $\mathcal{P}_{2}(T)$, where $R=R(\mathbf{T}, \mathbf{L})$ and $T=T(\mathbf{T})$ are as defined earlier and

- $\mathcal{P}_{1}(R)$ denotes the property that $R \cap E(H)$ does not contain a copy of $F_{1}$,

- $\mathcal{P}_{2}(T)$ denotes the property that for each $e \in T$ there exists a copy of $F_{1}$ in $H$ (denoted by $F_{e}$ ) such that $E\left(F_{e}\right) \cap E\left(F_{e^{\prime}}\right)=\emptyset$ for different $e, e^{\prime} \in T$.

Estimating the probability $H \nrightarrow\left(F_{1}, \ldots, F_{r}\right)$. Finally, for $H \sim H^{k}(n, p)$, we can upper bound the probability that $H \nrightarrow\left(F_{1}, \ldots, F_{r}\right)$ as follows (recall $\mathcal{F}=\mathcal{F}\left(F_{1}\right.$, $H)$ and $\left.\mathcal{I}=\mathcal{I}\left(F_{1}, H\right)\right)$ :

$$
\begin{aligned}
\operatorname{Pr}[H & \left.\nrightarrow\left(F_{1}, \ldots, F_{r}\right)\right] \\
& \leqslant \operatorname{Pr}\left[|\mathcal{F} \backslash \mathcal{I}|>n^{k-1 / m_{k}\left(F_{2}\right)-\delta}\right]+\operatorname{Pr}\left[\exists \mathbf{T}, \mathbf{L}: H \in \mathcal{P}_{1}(R(\mathbf{T}, \mathbf{L})) \cap \mathcal{P}_{2}(T(\mathbf{T}))\right] \\
& \leqslant o(1)+\sum_{\mathbf{T}} \sum_{\mathbf{L}} \operatorname{Pr}\left[H \in \mathcal{P}_{1}(R(\mathbf{T}, \mathbf{L})) \cap \mathcal{P}_{2}(T(\mathbf{T}))\right] .
\end{aligned}
$$

The first probability is $o(1)$ by Claim 16 . Note that $\mathcal{P}_{1}(R)$ is a decreasing and $\mathcal{P}_{2}(T)$ is an increasing graph property, thus by the FKG inequality (see for example, [1, Theorem 6.3.3]) we know they are negatively correlated, that is,

$\operatorname{Pr}\left[H \nrightarrow\left(F_{1}, \ldots, F_{r}\right)\right] \leqslant o(1)+\sum_{\mathbf{T}} \sum_{\mathbf{L}} \operatorname{Pr}\left[H \in \mathcal{P}_{1}(R(\mathbf{T}, \mathbf{L}))\right] \cdot \operatorname{Pr}\left[H \in \mathcal{P}_{2}(T(\mathbf{T}))\right]$.

Our aim is to show that the double sum in (7) also sums up to $o(1)$. 
For fixed choices of $\mathbf{T}$ and $\mathbf{L}$, and therefore for fixed $R$, from Lemma 13 and Claim 17 we get

$$
\operatorname{Pr}\left[\mathcal{P}_{1}(R)\right] \leqslant e^{-\beta C n^{k-1 / m_{k}\left(F_{2}\right)}},
$$

for some $\beta>0$. On the other hand, the expected number of mappings $T \rightarrow \mathcal{F}$ which are witnesses for the property $\mathcal{P}_{2}(T)$ is at most

$$
\left(n^{v\left(F_{1}\right)-k} p^{e\left(F_{1}\right)}\right)^{|T|}=C^{e\left(F_{1}\right)|T|} n^{-|T| / m_{k}\left(F_{2}\right)} .
$$

Consequently, the probability that $H$ has the property $\mathcal{P}_{2}(T)$ is at most this value. We can now upper bound the sum in (7) as

$$
\operatorname{Pr}\left[H \nrightarrow\left(F_{1}, \ldots, F_{r}\right)\right] \leqslant o(1)+e^{-\beta C n^{k-1 / m_{k}\left(F_{2}\right)}}\left(\sum_{\mathbf{T}} \sum_{\mathbf{L}} C^{e\left(F_{1}\right)|T|} n^{-|T| / m_{k}\left(F_{2}\right)}\right) .
$$

Next, from $m_{k}\left(F_{2}\right) \geqslant m_{k}\left(F_{i}\right)$ for $i \geqslant 3$ we observe that Theorem 15 implies that $\left|T^{i}\right| \leqslant \ell n^{k-1 / m_{k}\left(F_{2}\right)}$ for $i \geqslant 2$, where $\ell=\max _{i \geqslant 2} \ell_{i}$. Therefore, for each $t \in \mathbb{N}$ with $t \leqslant r \ell n^{k-1 / m_{k}\left(F_{2}\right)}$ (the maximal size of $T$ ) we can upper bound the number of choices of $\mathbf{T}$ by picking $t$ edges and for each $T^{i}=\left(T_{1}^{i}, \ldots, T_{\ell_{i}}^{i}\right) \in \mathcal{T}_{\ell_{i}}$ and each $j \in\left\{1, \ldots, \ell_{i}\right\}$ we decide which edges go to $T_{j}^{i}$. We can do this in at most

$$
\left(\begin{array}{c}
n^{k} \\
t
\end{array}\right) 2^{\ell r t}
$$

ways, where the $2^{\ell r t}$ factor comes from the upper bound on the number of ways how to distribute $t$ edges among $T_{j}^{i}$, s. On the other hand, we can choose each $L_{i}$ in at most

$$
\left(\begin{array}{c}
n^{k} \\
C^{\prime} n^{k-1 / m_{k}-\delta}
\end{array}\right) \leqslant n^{k \cdot C^{\prime} n^{k-1 / m_{k}\left(F_{2}\right)-\delta}}=e^{o\left(n^{k-1 / m_{k}\left(F_{2}\right)}\right)}
$$

ways, for some constant $C^{\prime}>0$, cf. (6). Using these estimates, we further bound the double sum in (8) as follows

$$
\sum_{\mathbf{T}} \sum_{\mathbf{L}} \cdots \leqslant e^{o\left(n^{k-1 / m_{k}\left(F_{2}\right)}\right)} \sum_{t=0}^{r \ell n^{k-1 / m_{k}\left(F_{2}\right)}}\left(\begin{array}{c}
n^{k} \\
t
\end{array}\right) 2^{r \ell t} C^{e\left(F_{1}\right) t} n^{-t / m_{k}\left(F_{2}\right)} .
$$

Using the estimate $\left(\begin{array}{l}n \\ \ell\end{array}\right) \leqslant(e n / \ell)^{\ell}$, we further simplify the sum on the right hand side,

$$
\begin{aligned}
\sum_{t=0}^{r \ell n^{k-1 / m_{k}\left(F_{2}\right)}}\left(\begin{array}{c}
n^{k} \\
t
\end{array}\right) 2^{r \ell t} C^{e\left(F_{1}\right) t} n^{-t / m_{k}\left(F_{2}\right)} & \leqslant \sum_{t=0}^{r \ell n^{k-1 / m_{k}\left(F_{2}\right)}}\left(\frac{e n^{k}}{t} 2^{r \ell} C^{e\left(F_{1}\right)} n^{-1 / m_{k}\left(F_{2}\right)}\right)^{t} \\
& \leqslant\left(r \ell n^{k-1 / m_{k}\left(F_{2}\right)}+1\right)\left(\frac{e 2^{r \ell} C^{e\left(F_{1}\right)}}{r \ell}\right)^{r \ell n^{k-1 / m_{k}\left(F_{2}\right)}} \\
& \leqslant\left(e 2^{r \ell} C^{e\left(F_{1}\right)}\right)^{r \ell n^{k-1 / m_{k}\left(F_{2}\right)}},
\end{aligned}
$$


where we used the fact that the maximal element of the sum is attained for $t=r \ell n^{k-1 / m_{k}\left(F_{2}\right)}$, since $g(t):=(m / t)^{t}$ is monotone increasing for $x \in[0, m / e]$. Finally, we obtain using (8):

$$
\operatorname{Pr}\left[H \nrightarrow\left(F_{1}, \ldots, F_{r}\right)\right] \leqslant o(1)+e^{-\beta C n^{k-1 / m_{k}\left(F_{2}\right)}} e^{o\left(n^{k-1 / m_{k}\left(F_{2}\right)}\right)}\left(e 2^{r \ell} C^{e\left(F_{1}\right)}\right)^{r \ell n^{k-1 / m_{k}\left(F_{2}\right)}},
$$

which is $o(1)$ for sufficiently large $C>0$.

\section{Asymmetric Ramsey properties: 0-statement}

In this section we prove Theorem 8 , which we repeat here for the convenience of the reader.

THEOREM. Let $F_{1}, \ldots, F_{r}$ be $k$-uniform hypergraphs such that $F_{2}$ has at least three edges, $m_{k}\left(F_{1}\right) \geqslant m_{k}\left(F_{2}\right) \geqslant \cdots \geqslant m_{k}\left(F_{r}\right)>0$ and

(i) $F_{1}$ and $F_{2}$ are strictly balanced and $m_{2}\left(F_{2}\right) \geqslant 1$;

(ii) $F_{1}$ is strictly balanced with respect to $m_{k}\left(\cdot, F_{2}\right)$;

(iii) $\mathcal{F}^{*}\left(F_{1}, F_{2}\right)$ is asymmetric-balanced;

(iv) for every hypergraph $G$ such that $m(G) \leqslant m_{k}\left(F_{1}, F_{2}\right)$ it follows that

$$
G \nrightarrow\left(F_{1}, F_{2}\right) .
$$

Then there exists a constant $c>0$ such that for $p \leqslant c n^{-1 / m_{k}\left(F_{1}, F_{2}\right)}$

$$
\lim _{n \rightarrow \infty} \operatorname{Pr}\left[H^{k}(n, p) \rightarrow\left(F_{1}, \ldots, F_{r}\right)\right]=0 .
$$

Note that it is sufficient to prove the statement for the case of two colours. By having more than two colours we can always restrict to only the first two, which avoids a monochromatic copies of $F_{i}$ for $i \geqslant 3$. Thus we assume $r=2$ and we call the colours red and blue.

We use a grow-sequences approach very similar to the one in $[17,18]$. We say an edge is closed if it is contained in a copy of $F_{1}$ and a copy of $F_{2}$ which are otherwise edge-disjoint, and open otherwise. The reason for this distinction is that open edges are easy to colour. Assume $e$ is an open edge and there exists a valid 2-colouring (that is, one without red copy of $F_{1}$ and blue copy of $F_{2}$ ) for $H-e$, the hypergraph obtained from $H$ by removing $e$ from the edge set. Then we can extend this colouring to $H$ by using the fact that $e$ is open: if there exists a copy of $F_{1}$ in $H$, say $\hat{F}_{1}$, which contains $e$ and is already red up to $e$ then colour 
$e$ with blue, and otherwise colour it with red. This clearly avoids a red copy of $F_{1}$ in $H$. Let us assume, towards a contradiction, that there exists a blue copy of $F_{2}$. First, note that any such copy has to contain $e$ and therefore $e$ has to be coloured with blue. However, this implies that such copy of $F_{2}$ does not intersect $\hat{F}_{1}$ on any other edge except $e$ (since every other edge of $\hat{F}_{1}$ is red), which contradicts the assumption that $e$ is open.

Using the notion of an open edge, we can find a valid 2-colouring of $H^{k}(n, p)$ by running the following algorithm:

$$
\begin{aligned}
& \hat{H}:=H^{k}(n, p) \\
& \text { while } \hat{H} \text { contains an open edge e do } \\
& \mid \hat{H} \leftarrow \hat{H}-e \\
& \text { end }
\end{aligned}
$$

Colour $\hat{H}$ without a red $F_{1}$ and a blue $F_{2}$

Add the removed edges in reverse order and colour them appropriately.

Algorithm 1: Colouring algorithm.

Note that the obtained graph $\hat{H}$ is uniquely defined. Indeed, since if an edge is open in $H$ then it is also open in every $H^{\prime} \subseteq H$, the order in which we remove open edges is irrelevant.

Of course, the step 'Colour $\hat{H}$ ' in the Algorithm 1 is the difficult one and the main part of the proof is to show that this is indeed possible with high probability. Our strategy is to first show that $\hat{H}$ can be split into hypergraphs of constant size which can be coloured separately (using properties (i)-(iii)). Then using the bound on $p$ we deduce that every such hypergraph has small density and, finally, from the assumption (iv) we conclude that it can be coloured without red $F_{1}$ or blue $F_{2}$. To state this precisely we need a couple of definitions.

Definition $18\left(\left(F_{1}, F_{2}\right)\right.$-core). We say a subgraph $G^{\prime} \subseteq \hat{H}$ (where $\hat{H}$ is the resulting graph obtained by the Algorithm 1$)$ is an $\left(F_{1}, F_{2}\right)$-core if every copy of $F_{1}$ or $F_{2}$ in $\hat{H}$ is either contained in $G^{\prime}$ or edge-disjoint from $G^{\prime}$.

Since $\hat{H}$ is closed by the definition of the algorithm, every $\left(F_{1}, F_{2}\right)$-core is closed as well. However, to emphasize this fact we shall sometimes explicitly write that the $\left(F_{1}, F_{2}\right)$-core under consideration is closed.

Next, let $\hat{H}_{1}, \ldots, \hat{H}_{t}$ be a partition of $\hat{H}$ into edge-disjoint minimal (by subgraph inclusion) closed $\left(F_{1}, F_{2}\right)$-cores. By the definition of the core and the assumption that $\hat{H}_{i}$ 's are edge-disjoint, if there exists a valid colouring of each $\hat{H}_{i}$ 
then the same colourings induce a valid colouring of $\hat{H}_{1} \cup \cdots \cup \hat{H}_{t}=\hat{H}$. The following lemma is the key ingredient in the proof of Theorem 8.

Lemma 19. Let $F_{1}$ and $F_{2}$ be as in Theorem 8. Then there exist constants $c=$ $c\left(F_{1}, F_{2}\right)>0$ and $L=L\left(F_{1}, F_{2}\right)>0$ such that if $p \leqslant c n^{-1 / m_{k}\left(F_{1}, F_{2}\right)}$ then w.h.p. every minimal closed $\left(F_{1}, F_{2}\right)$-core of $H^{k}(n, p)$ has size at most $L$.

Before we go into the proof of the lemma, we first use it to derive Theorem 8 .

Proof of Theorem 8. Let $c$ and $L$ be as given by Lemma 19. First, note that w.h.p. every hypergraph $G \subset H^{k}(n, p)$ of size at most $L$ satisfies $m(G) \leqslant m_{k}\left(F_{1}, F_{2}\right)$. This can be derived as follows. For every hypergraph $G$ with at most $L$ vertices and $m(G)>m_{k}\left(F_{1}, F_{2}\right)$ we have

$$
1 / m_{k}\left(F_{1}, F_{2}\right) \geqslant 1 / m(G)+\alpha,
$$

for some $\alpha>0$. Moreover, there exists $\alpha=\alpha(L)$ such that this holds for all such hypergraphs $G$ simultaneously. Consider some $G$ with $m(G)>m_{k}\left(F_{1}, F_{2}\right)$ and let $G^{\prime} \subseteq G$ be such that $m(G)=e\left(G^{\prime}\right) / v\left(G^{\prime}\right)$. Note that the expected number of copies of $G^{\prime}$ in $H^{k}(n, p)$ is at most

$$
n^{v\left(G^{\prime}\right)} p^{e\left(G^{\prime}\right)} \leqslant n^{v\left(G^{\prime}\right)}\left(c n^{-1 / m_{k}\left(F_{1}, F_{2}\right)}\right)^{e\left(G^{\prime}\right)} \leqslant n^{v\left(G^{\prime}\right)} n^{-v\left(G^{\prime}\right)-\alpha}=n^{-\alpha} .
$$

Therefore, by Markov's inequality we have that $G^{\prime}$, and therefore $G$, does not appear in $H^{k}(n, p)$ with probability at least $n^{-\alpha / 2}$. Since there are at most $L 2^{L^{2}}$ such hypergraphs and $L$ does not depend on $n$, by union bound we have that w.h.p. none of them appears.

Next, let $\hat{H}$ be the hypergraph obtained using the Algorithm 1 and let $\hat{H}_{1}$, $\ldots, \hat{H}_{t}$ be a partition of $\hat{H}$ into edge-disjoint minimal closed $\left(F_{1}, F_{2}\right)$-cores. By Lemma 19 we have that each $\hat{H}_{i}$ has size at most $L$, and from the previous observation we conclude that $m\left(\hat{H}_{i}\right) \leqslant m_{k}\left(F_{1}, F_{2}\right)$. Now using the property (iv) we obtain a colouring of $\hat{H}_{i}$ without a monochromatic $F_{1}$ and $F_{2}$ which, by the discussion preceding the proof, gives a valid colouring of $\hat{H}$. This, in turn, gives a valid colouring of the whole $H^{k}(n, p)$ which finishes the proof.

In the rest of this section we prove Lemma 19. The proof of the lemma will rely on a somewhat technical claim which we postpone to the next section.

Proof of Lemma 19. Our strategy is to show that every minimal closed $\left(F_{1}, F_{2}\right)$ core is either of size at most $L$ or is much larger, namely of size $\Omega(\log n)$. Then, using some further properties of such hypergraphs, we deduce that the latter case does not happen in $H^{k}(n, p)$. Our main tool in proving this is a procedure (Algorithm 2) which generates each $\left(F_{1}, F_{2}\right)$-core in a systematic way. 
1 Let $F_{1}^{*}$ be a copy of $F_{1}$ in $G$

$2 G_{1} \leftarrow F_{1}^{*}$

3 $i \leftarrow 1$

4 while $G_{i} \neq G$ do

5 $\quad i \leftarrow i+1$

$6 \quad$ if $G_{i-1}$ contains an open edge then

$7 \quad j \leftarrow$ smallest index $j<i$ such that $F_{j}^{*}$ contains open edges

$8 \quad e \leftarrow$ the minimal open edge in $F_{j}^{*}$

9

if there exists a copy $\hat{F}_{1}$ of $F_{1}$ in $G$, not contained in $G_{i-1}$, which contains $e$ then

$F_{i}^{*} \leftarrow \hat{F}_{1}$

else

$F_{i}^{*} \leftarrow$ a copy of some hypergraph from $\mathcal{F}^{*}$ in $G$ but not in $G_{i-1}$, which contains $e$ as an attachment edge

\section{end} else

if there exists a copy $\hat{F}_{1}$ of $F_{1}$ in $G$, not contained in $G_{i-1}$, which intersects $G_{i-1}$ in at least one edge then

$F_{i}^{*} \leftarrow \hat{F}_{1}$

\section{else}

$F_{i}^{*} \leftarrow$ a copy $F^{*}$ of a hypergraph in $\mathcal{F}^{*}$ in $G$, not contained in

$G_{i-1}$, such that its attachment edge is contained in $G_{i-1}$

end

end

$G_{i} \leftarrow G_{i-1} \cup F_{i}^{*}$

Algorithm 2: Decomposing minimal closed $\left(F_{1}, F_{2}\right)$-cores.

Let $G$ be some minimal closed $\left(F_{1}, F_{2}\right)$-core. We assume that some arbitrary total ordering on the vertices of $G$ is given. By lexicographic ordering this induces a total ordering on the edges of $G$ as well, that is, we can always choose a well-defined minimal edge out of any edge set. For the enumeration aspect of the problem we map any minimal closed $\left(F_{1}, F_{2}\right)$-core $G$ to a sequence of hypergraphs $F_{i}^{*}$ 's via Algorithm 2, where $F_{i}^{*}$ is either a member of $\mathcal{F}^{*}\left(F_{1}, F_{2}\right)$ (see Definition 6) or is isomorphic to $F_{1}$. We denote the family $\mathcal{F}^{*}\left(F_{1}, F_{2}\right)$ by $\mathcal{F}^{*}$, as it will always be used with respect to $F_{1}$ and $F_{2}$.

Let us first prove that Algorithm 2 is well defined. If $G_{i-1}$ has an open edge $e$, then either there is a copy of $F_{1}$ or $F_{2}$ not contained in $G_{i-1}$, but which contains $e$. 
Moreover, note that every copy of $F_{2}$ is contained in some $F^{*} \in \mathcal{F}\left(F_{1}, F_{2}\right)$, since each edge of $G$ is closed. These observations prove that if $G_{i-1}$ has an open edge, then one of the two requirements of the lines 9 and 12 are satisfied.

Similarly, if $G_{i-1}$ does not have an open edge and $G_{i-1} \neq G$ then there is a copy of $F_{1}$ or $F_{2}$ which intersects $G_{i-1}$, but which is not contained in it. By the previous argument, one of the two requirements of the lines 15 and 18 are satisfied. The algorithm is therefore correct and terminates.

Note that the sequence $S:=\left(F_{1}^{*}, \ldots, F_{\ell}^{*}\right)$ fully describes a run of the algorithm. We call it a grow sequence for $G$ and each $F_{i}^{*}$ in it a step of the sequence, where $1 \leqslant i \leqslant \ell$. Set $S_{i}=\left(F_{1}^{*}, \ldots, F_{i}^{*}\right)$ to be the grow sequence consisting of the first $i$ steps. With this we turned the problem of enumerating all minimal closed $\left(F_{1}, F_{2}\right)$ cores into the one of enumerating all grow sequences which may appear as output of Algorithm 2. We aim to estimate the expected number of grow sequences. As already hinted in the beginning of the proof, we show that the algorithm either terminates with a grow sequence of size $O(1)$, or it has to produce a sequence $S$ of size $\Omega(\log n)$. However, in the latter case we show that a subsequence of $S$ truncated after the first $\Theta(\log n)$ steps does not appear in $H^{k}(n, p)$, which in turn implies that $S$ does not appear either. We now make this precise.

We distinguish various step types. $F_{1}^{*}$ is the first step. Steps chosen in lines 12 and 18 are called regular if $\left.e\left(F_{i}^{*}\right)-e\left(H_{i}\right)\right) /\left(v\left(F_{i}^{*}\right)-v\left(H_{i}\right)\right)=m_{k}\left(F_{1}, F_{2}\right)$, where $H_{i}:=F_{i}^{*} \cap G_{i-1}$, otherwise they are called degenerate. Furthermore, regular step is open or closed, depending whether it was chosen in line 12 or 18 . The steps chosen in lines 9 and 15 are by definition always degenerate. We say $F_{1}^{*}$ is degenerate by definition.

Consider any open regular step $F_{i}^{*}$. By asymmetric-balancedness of $\mathcal{F}^{*}$, its intersection $H_{i}$ with $G_{i-1}$ is exactly one edge and $F_{i}^{*}$ is generic, and thus we can bound the contribution of each such step to the expected number of sequences by

$$
k ! \cdot n^{v\left(F_{i}^{*}\right)-k} p^{e\left(F_{i}^{*}\right)-1} .
$$

By using $p \leqslant c n^{-1 / m_{k}\left(F_{1}, F_{2}\right)}$ and assumption (iii) from Theorem 8 we obtain

$$
n^{v\left(F_{i}^{*}\right)-k} p^{e\left(F_{i}^{*}\right)-1} \leqslant c^{e\left(F_{i}^{*}\right)-1} n^{v\left(F_{i}^{*}\right)-k-\left(e\left(F_{i}^{*}\right)-1\right) / m_{k}\left(F_{1}, F_{2}\right)} \leqslant c^{e\left(F_{i}^{*}\right)-1} .
$$

It follows that

$$
k ! \cdot n^{v\left(F_{i}^{*}\right)-k} p^{e\left(F_{i}^{*}\right)-1} \leqslant k ! \cdot c^{e\left(F_{i}^{*}\right)-1} \leqslant c,
$$

where the last inequality holds as we may choose the constant $c$ small enough, and such that $\log (c)<-1$ holds.

At every step, we add some new vertices, but never more than the number of vertices in some generic $F^{*}$. That means that after $i-1$ steps there are at most 
$v\left(F^{\$}\right) \cdot(i-1)$ vertices in $G_{i-1}$. Hence, if $F_{i}^{*}$ is a closed regular step, we can bound the contribution to the expected number of sequences by

$$
k !\left(v\left(F^{8}\right) \cdot(i-1)\right)^{k} n^{v\left(F_{i}^{*}\right)-k} p^{e\left(F_{i}^{*}\right)-1} \leqslant c\left(v\left(F^{\$ 8}\right) \cdot i\right)^{k} .
$$

Now consider the case of a degenerate step $F_{i}^{*}$ which is a copy of some $F^{*} \in$ $\mathcal{F}^{*}$. By the asymmetric-balancedness condition we can choose a constant $\alpha_{1}>0$ such that regardless of the choice of $F^{*} \in \mathcal{F}^{*}$ we have (recall that $H_{i}=F_{i}^{*} \cap G_{i-1}$ )

$$
v\left(F^{*}\right)-v\left(H_{i}\right)-\frac{e\left(F^{*}\right)-e\left(H_{i}\right)}{m_{k}\left(F_{1}, F_{2}\right)}<-\alpha_{1} .
$$

In the case of a degenerate step consisting of just a copy of $F_{1}$ (that is, one as in line 9 or 15) we can choose $\alpha_{2}>0$ such that for all $H_{i} \subsetneq F_{1}, e\left(H_{i}\right) \geqslant 1$, we have

$$
v\left(F_{1}\right)-v\left(H_{i}\right)-\frac{e\left(F_{1}\right)-e\left(H_{i}\right)}{m_{k}\left(F_{1}, F_{2}\right)}<-\alpha_{2},
$$

by the fact that $F_{1}$ is strictly balanced with respect to $m_{k}\left(\cdot, F_{2}\right)$. Note that this holds even for $H_{i}$ being exactly one edge, as $m_{k}\left(F_{1}\right)>m_{k}\left(F_{1}, F_{2}\right)$. We then set $\alpha=\min \left\{\alpha_{1}, \alpha_{2}\right\}$.

With this we obtain an upper bound for the contribution of a degenerate step $F_{i}^{*}$,

$$
\left|\mathcal{F}^{*}\right| \cdot v\left(F_{i}^{*}\right)^{v\left(H_{i}\right)}\left(i \cdot v\left(F^{\&}\right)\right)^{v\left(H_{i}\right)} \cdot n^{v\left(F_{i}^{*}\right)-v\left(H_{i}\right)} p^{e\left(F_{i}^{*}\right)-e\left(H_{i}\right)} \leqslant C i^{v\left(F^{88}\right)} n^{-\alpha},
$$

where $C$ is a suitably chosen constant.

To finish the argument we use the following claim whose proof is presented in the next section.

Claim 20. There exist positive constants $C_{1}, C_{2}$ (depending on $F_{1}$ and $F_{2}$ ) such that the following holds. Let $S$ be a grow sequence of length $t$.

(i) If $S$ contains at most d degenerate steps, then $t \leqslant d\left(1+C_{2} / C_{1}\right)$.

(ii) If a prefix $S_{i}$ of $S$ contains at most d degenerate steps, then $S_{i}$ contains no closed regular steps $F_{j}$ with $j>d\left(1+C_{2} / C_{1}\right)+1$.

Let $C_{1}, C_{2}$ be as in the claim above. We can now finish our first moment argument. Set $d_{\max }:=v\left(F_{1}\right) / \alpha+1$ and $L=\left(1+C_{2} / C_{1}\right) \cdot d_{\max }+1$. By Claim 20 all sequences longer than $L$ must contain at least $d_{\max }$ degenerate steps. Set $t_{\max }:=v\left(F_{1}\right) \log (n)+d_{\max }+1$. We consider two cases: the first are those sequences which have their $d_{\max }$ th degenerate step before $t_{\max }$. We truncate them 
after the $d_{\max }$ th step. The second are those sequences whose $d_{\max }$ th step appears after $t_{\max }$. We truncate these at length $t_{\max }$. Note that by Claim 20 in both of these cases closed regular steps can only happen in the first $L$ steps of the sequence.

Let us now analyse the first case when $d_{\max }$ th degenerate step happens before $t_{\max }$. Using (12) and (13) and summing over all possible steps $t$ when $d_{\max }$ th degenerate step happened we bound the expected number of such grow sequences

$$
\begin{aligned}
& \sum_{t=L+1}^{t_{\max }-1} n^{v\left(F_{1}\right)}\left(\begin{array}{c}
{ }^{t} \\
d_{\max }
\end{array}\right)\left(C t^{v\left(F^{\$ 8}\right)} n^{-\alpha}\right)^{d_{\max }}\left(v\left(F^{\& 8}\right) L\right)^{k L}= \\
& \quad=\mathcal{O}\left(\operatorname{polylog}(n) \cdot n^{v\left(F_{1}\right)} n^{-\alpha \cdot d_{\max }}\right)=\mathcal{O}\left(\operatorname{polylog}(n) \cdot n^{v\left(F_{1}\right)-v\left(F_{1}\right)-\alpha}\right)=o(1) .
\end{aligned}
$$

Here we bound the contribution of the first step by $n^{v\left(F_{1}\right)}$, drop the contribution of $c<1$ for all regular steps, and use the fact that only the first $L$ steps may be closed regular.

In the second case, by summing over the number of degenerate steps $d$ before step $t_{\max }$, we obtain

$$
\begin{aligned}
& \sum_{d=0}^{d_{\max }} n^{v\left(F_{1}\right)}\left(\begin{array}{c}
t_{\max } \\
d
\end{array}\right)\left(C t_{\max }^{v\left(F^{8}\right)} n^{-\alpha}\right)^{d}\left(v\left(F^{8}\right) L\right)^{k L} c^{t_{\max }-d-1} \\
& \quad=\mathcal{O}\left(\operatorname{polylog}(n) \cdot n^{v\left(F_{1}\right)} c^{t_{\max }-1-d}\right)=\mathcal{O}\left(\operatorname{polylog}(n) \cdot n^{v\left(F_{1}\right)(1+\log c)}\right)=o(1),
\end{aligned}
$$

where the last step holds because $\log c<-1$. With this we proved that only grow sequences of length at most the constant $L$ can appear in $H^{k}(n, p)$ for $p \leqslant$ $c n^{1 / m_{k}\left(F_{1}, F_{2}\right)}$.

It remains to prove Claim 20, which we do in the next section.

4.1. Bounding grow sequences-Proof of Claim 20. Let us first prove an auxiliary claim.

Claim 21. Let $G$ be an arbitrary hypergraph and let $F_{1}, F_{2}$ be as in Theorem 8. Furthermore, let $F$ be an $F_{1}$-copy which intersects $G$ in exactly one edge $e$ in $E(G)$. Set $G_{F}$ to be a hypergraph $(V(G) \cup V(F), E(G) \cup E(F))$. Then every edge in $E(F) \backslash$ e is open in $G_{F}$.

Proof. Let $e^{*}$ be an arbitrary edge from $E(F) \backslash e$. We first prove that any $F_{1}$-copy that contains $e^{*}$, must contain all edges from $E(F) \backslash e$. Note that this is equivalent to proving that all $F_{1}$-copies that contain $e^{*}$ have exactly one edge intersecting $e(G)$. In order to arrive at a contradiction let $F^{\prime}$ be an $F_{1}$-copy such that 
$\left|E\left(F^{\prime}\right) \cap E(G)\right| \geqslant 2$. Set $F_{\text {new }}=F^{\prime}[(V(F) \backslash V(G)) \cup e]$ and $F_{\text {old }}=F^{\prime}[V(G)]$. Furthermore, let us denote $F_{\text {new }}^{+e}$ as the hypergraph obtained by adding edge $e$ to $F_{\text {new }}$ (if the edge is already in $F_{\text {new }}$ then $F_{\text {new }}=F_{\text {new }}^{+e}$ ). Note that both $F_{\text {new }}^{+e}$ and $F_{\text {old }}$ are strict subgraphs of $F_{1}$. Since $e\left(F^{\prime}\right)=e\left(F_{\text {new }}^{+e}\right)+e\left(F_{\text {old }}\right)-1$ and $v\left(F^{\prime}\right) \geqslant v\left(F_{\text {new }}^{+e}\right)+v\left(F_{\text {old }}\right)-k$ we obtain

$$
m_{\ell}\left(F^{\prime}\right)=\frac{e\left(F^{\prime}\right)-1}{v\left(F^{\prime}\right)-k} \leqslant \frac{e\left(F_{\text {new }}^{+e}\right)-1+e\left(F_{\text {old }}\right)-1}{v\left(F_{\text {new }}^{+e}\right)-k+v\left(F_{\text {old }}\right)-k} .
$$

Since $F^{\prime}$ is strictly balanced we know that $m_{k}\left(F_{\text {new }}^{+e}\right)$ and $m_{k}\left(F_{\text {old }}\right)$ are strictly smaller than $m_{k}\left(F^{\prime}\right)$. Together with inequality above, this implies

$$
m_{k}\left(F^{\prime}\right) \leqslant \frac{e\left(F_{\text {new }}^{+e}\right)-1+e\left(F_{\text {old }}\right)-1}{v\left(F_{\text {new }}^{+e}\right)-k+v\left(F_{\text {old }}\right)-k}<m_{k}\left(F^{\prime}\right),
$$

which is a contradiction.

In order to prove the lemma, it is sufficient to prove that any $F_{2}$-copy that contains $e^{*}$ must intersect $E(F) \backslash e$ on at least one edge other than $e^{*}$. Assume $H$ is an $F_{2}$-copy which contains $e^{*}$ but otherwise is fully contained in $G$. Let us denote $H_{\text {old }}$ be $H[V(G)]$. Since $H \cong F_{2}$ is strictly $m_{k}$-balanced, we have $m_{k}(H)>\left(e\left(H_{\text {old }}\right)-1\right) /\left(v\left(H_{\text {old }}\right)-k\right)$. Therefore we obtain with $m_{k}(H) \geqslant 1$ that

$$
m_{k}(H)>\frac{e\left(H_{\text {old }}\right)-1+1}{v\left(H_{\text {old }}\right)-k+1} .
$$

This is, however, a contradiction as $m_{k}(H)=(e(H)-1) /(v(H)-k)=$ $e\left(H_{\text {old }}\right) /\left(v\left(H_{\text {old }}\right)+\left(v(H)-v\left(H_{\text {old }}\right)\right)-k\right)$ and $H$ contains at least one vertex more than $H_{\text {old }}$. We conclude that any $F_{1}$-copy and $F_{2}$-copy that contain $e^{*}$ intersect on at least two edges and thus $e^{*}$ is closed.

Before we continue with the proof of Claim 20, we introduce some definitions. For any regular step $F_{i}^{*}$, we call the edge $e=E\left(G_{i-1}\right) \cap E\left(F_{i}^{*}\right)$ the attachment edge of $F_{i}^{*}$ and the vertices in $V\left(F_{i}^{*}\right) \backslash V\left(G_{i-1}\right)$ the inner vertices of $F_{i}^{*}$. If $F_{i}^{*}$ is a regular step, then $F_{i}^{*}$ contains $\left(e\left(F_{2}\right)-1\right)\left(e\left(F_{1}\right)-1\right)$ open edges in $G_{i}$ (that is, every edge in a petal of $F_{i}^{*}$, not contained in the centre of $F_{i}^{*}$ ). In fact, we call a step $F_{j}^{*}, j \leqslant i$, fully open in $S_{i}$ if it is a regular step and no other step $F_{j}^{*}$, $j<j^{\prime} \leqslant i$, intersects any vertex of $F_{j}^{*}$ which is not in the attachment edge of $F_{j}^{*}$. Note that by Claim 21 a fully open step $F_{j}^{*}$ in $S_{i}$ contains $\left(e\left(F_{2}\right)-1\right)\left(e\left(F_{1}\right)-1\right)$ open edges in $G_{i}$. The next claim states that a fully open step $F_{i}^{*}$ stays fully open if none of the following steps intersects its inner vertices.

Claim 22. Let $S=\left(F_{1}^{*}, \ldots, F_{l}^{*}\right)$ be a grow sequence and $F_{j}^{*}, j \leqslant i$ a regular step, for some $i \leqslant l$. Then if no step $F_{j^{\prime}}^{*}$, for $j^{\prime} \in\{j+1, \ldots, i\}$, contains an inner vertex of $F_{j}^{*}$, then $F_{j}^{*}$ is fully open in $S_{i}$. 
Proof. Let $G_{i}$ be the graph obtained at $i$ th step of the grow sequence and let $F_{2}$ be the 'centre' and $F_{1}^{1}, \ldots, F_{1}^{e\left(F_{2}\right)-1}$ be the 'petals' of $F_{j}^{*}$. Set

$$
H_{j}:=G_{i-1} \cup F_{2} \cup \bigcup_{t \in\left[e\left(F_{2}\right)-1\right] \backslash\{j\}} F_{1}^{t} .
$$

By applying Claim 21 to $H_{t}$ (as $G$ ) and $F_{1}^{t}$ as $(F)$ for all $t \in\left[e\left(F_{2}\right)\right]$ we obtain the lemma.

For $i \geqslant 1$ let $\kappa(i)$ denote the number of fully open copies 'destroyed' by step $i$, that is,

$$
\kappa(i)=\mid\left\{j<i: F_{j}^{*} \text { is fully open in } S_{i-1} \text { but not in } S_{i}\right\} \mid .
$$

The following claims show how regular and degenerate steps influence $\kappa(\cdot)$.

CLAIM 23. If $F_{i}^{*}$ is a regular step, then $\kappa(i) \leqslant 1$.

Proof. Since the attachment edge of $F_{i}^{*}$ can intersect inner vertices of only one regular step, together with Claim 22 the proof is concluded.

Claim 24. If $F_{i}^{*}$ is a degenerate step, then $\kappa(i) \leqslant v\left(F_{i}^{*}\right)$.

Proof. Let us first prove that any vertex from $G_{i-1}$ is an inner vertex of at most one regular copy $F_{j}^{*}$ which is fully open in $S_{i-1}$. Let $v \in G_{i-1}$ be an arbitrary vertex from $G_{i-1}$ which is an inner vertex of some regular copy $F_{j}^{*}$ such that $F_{j}^{*}$ is fully open in $S_{i-1}$. By definition of a fully open copy, no step $F_{t}^{*}$, for $j<t<i$, can contain $v$. On the other hand, $v \notin G_{j-1}$ as $v$ is an inner vertex of a regular step.

By Claim 22, a fully open step $F_{j}^{*}$ in $S_{i-1}$ is a fully open step in $S_{i}$ if $F_{i}^{*}$ does not intersect inner vertices of $F_{j}^{*}$. This together with the observation above proves the claim.

Claim 25. Set $d=\left(e\left(F_{2}\right)-2\right)$. Let $F_{i}^{*}, \ldots, F_{i+d}^{*}$ be a sequence of consecutive regular steps such that $\kappa(i)=1$. Then $\kappa(i+1)=\cdots=\kappa(i+d)=0$.

Proof. As $\kappa(i)=1$ it holds that $F_{i}^{*}$ is the first step which intersects the inner vertices of some fully open step $F_{j}^{*}, j<i$. From previous observations we have that $F_{j}^{*}$ has $\left(e\left(F_{2}\right)-1\right)\left(e\left(F_{1}\right)-1\right)$ open edges before the step $F_{i}^{*}$ is made. It is sufficient to prove that after steps $F_{i}^{*}, \ldots, F_{i+s}^{*}$, for $s<e\left(F_{2}\right)-2$, there is still at least one open edge in $F_{j}^{*}$. Let $F_{1}^{\prime}, \ldots, F_{e\left(F_{2}\right)-1}^{\prime}$ be the petals of $F_{j}^{*}$ (which are copies of $F_{1}$ ). Note that $F_{i+1}^{*}, \ldots, F_{i+s}^{*}$ can intersect at most $s$ of different petals and without loss of generality let us assume they are $F_{1}^{\prime}, \ldots, F_{q}^{\prime}$, for $q \leqslant s$. 
By applying Claim 21 to $F_{q+1}^{\prime}$ (as $F$ ) and $G_{i+s}$ (as $G$ ) we obtain that all the edges in $F_{q+1}^{\prime}$, except for maybe one, are open in $G_{i+s}$. Observe that this is the case since we always choose 'minimal' open edges (cf. Algorithm 2) and for two fully open steps in some $S_{i}$ those edges are smaller that appear first in the grow sequence.

Using the previous three claims we are able to show a connection between the number of fully open steps in $S_{i}$ and the number of regular and degenerate steps in $S_{i}$. To simplify notation, we denote by $\operatorname{reg}\left(S_{i}\right), \operatorname{deg}\left(S_{i}\right)$ and fo $\left(S_{i}\right)$ the number of regular, degenerate and fully open steps in $S_{i}$, respectively.

Claim 26. Set $C_{1}:=1-1 /\left(e\left(F_{2}\right)-2\right)$ and $C_{2}:=v\left(F_{2}\right)+\left(e\left(F_{2}\right)-1\right)\left(v\left(F_{1}\right)-\right.$ $k)+1$. Then $\operatorname{fo}\left(S_{i}\right) \geqslant \operatorname{reg}\left(S_{i}\right) \cdot C_{1}-\operatorname{deg}\left(S_{i}\right) \cdot C_{2}$.

Proof. Set $\varphi(i)=\operatorname{fo}\left(S_{i}\right)-\operatorname{reg}\left(S_{i}\right) \cdot C_{1}+\operatorname{deg}\left(S_{i}\right) \cdot C_{2}$. We prove by induction the following, stronger, claim: for every $i \geqslant 1$

$$
\varphi(i) \geqslant \begin{cases}1 & F_{i}^{*} \text { is a degenerate step } \\ 0 & F_{i}^{*} \text { is a regular step. }\end{cases}
$$

Since the first step is by definition degenerate the hypothesis is true for $i=1$. Assume the claim holds for all $i<i^{\prime}$. If $\kappa\left(i^{\prime}\right)=0$, then since $C_{1}<1$ and $C_{2} \geqslant 1$ one can easily check that the claim holds. Thus, let us assume $\kappa\left(i^{\prime}\right)>0$. Furthermore, if $F_{i^{\prime}}^{*}$ is a degenerate step then by Claim 24 we know $\varphi\left(i^{\prime}\right) \geqslant$ $\varphi\left(i^{\prime}-1\right)+1$. From the induction hypothesis, this implies $\varphi\left(i^{\prime}\right) \geqslant 1$.

From now on we assume $\kappa\left(i^{\prime}\right)=1$ and $F_{i^{\prime}}^{*}$ is a regular step. Let $j<i^{\prime}$ be the largest integer such that $\kappa(j)>0$ or that $F_{j}^{*}$ is a degenerate step. One easily obtains

$$
\varphi\left(i^{\prime}\right)=\varphi(j)+i^{\prime}-j-1-\left(i^{\prime}-j\right) C_{1} .
$$

If $F_{j}^{*}$ is a degenerate step, then $\varphi(j) \geqslant 1$ and by (14) and $C_{1} \leqslant 1$ we have $\varphi\left(i^{\prime}\right) \geqslant$ 0 . Let us consider the case when $F_{j}^{*}$ is a regular step. By Claim 25 we know that $i^{\prime}-j \geqslant e\left(F_{2}\right)-1$. Thus

$$
\varphi\left(i^{\prime}\right)=\varphi(j)+(i-j)\left(1-C_{1}\right)-1>\varphi(j)+1-1 \geqslant 0,
$$

where the last inequality holds as $F_{2}$ has at least three edges. This concludes the proof.

We have all the necessary tools to finish the proof of Claim 20. Let $C_{1}, C_{2}$ be as in Claim 26. Let $S$ be any grow sequence of length $t$, and $S_{i}, i \leqslant t$ such that $i=t$ or $F_{i+1}^{*}$ is a closed regular step. Furthermore, let us assume $S_{i}$ contains at most $d$ 
degenerate steps. By construction, graph $G_{i}$ does not have an open edge and thus it does not have a fully open copy, that is, $f \circ\left(S_{i}\right)=0$. Using Claim 26 we obtain

$$
\text { fo }\left(S_{i}\right)=0 \geqslant \operatorname{reg}\left(S_{i}\right) \cdot C_{1}-\operatorname{deg}\left(S_{i}\right) \cdot C_{2} .
$$

Since there are at most $d$ degenerate steps in $S_{i}$, by using the previous observation we obtain

$$
d C_{2} \geqslant(i-d) C_{1},
$$

which implies $i \leqslant d\left(1+C_{2} / C_{1}\right)$. This concludes the proof of Claim 20 .

\section{Threshold for $H^{k}(n, p) \rightarrow\left(K_{3}^{+k}, C_{t_{k}}\right)$}

In this section we prove Lemma 10, one of the ingredients in the proof of Theorem 3. We do that by applying Theorem 8. Since it is easy to check that property $(i)$ in Theorem 8 holds and that $K_{3}^{+k}$ is strictly balanced with respect to $m_{k}\left(\cdot, C_{t_{k}}\right)$, in the next two sections we verify properties (iii) and (iv).

5.1. Property (iv). In this section we prove that property (iv) of Theorem 8 holds for $F_{1}=K_{3}^{+k}$ and $F_{2}=C_{t_{k}}$. For convenience we state this as a lemma.

LEMMA 27. For $k \geqslant 4$ and every $k$-uniform hypergraph $H$ such that $m(H) \leqslant$ $m_{k}\left(K_{3}^{+k}, C_{t_{k}}\right)$ we have

$$
H \nrightarrow\left(K_{3}^{+k}, C_{t_{k}}\right) \text {. }
$$

First we introduce some notation and definitions. We denote with $T_{\ell}^{k}$ a $k$ uniform hypergraph on the vertex set $\left\{v_{1}, \ldots, v_{\ell}\right\}$ and the edge set given by $\left\{v_{i}\right.$, $\left.\ldots, v_{i+k-1}\right\}$ for each $1 \leqslant i \leqslant \ell-k+1$. We refer to $T_{\ell}^{k}$ as a tight path of size $\ell$. An ordering $\left(e_{1}, \ldots, e_{\ell-k+1}\right)$ of the edges of $T_{\ell}^{k}$ is called natural if $\left|e_{i} \cap e_{i+1}\right|=k-1$ for every $1 \leqslant i \leqslant \ell-k$. Finally, we say that a set $S$ of hyperedges is $s$-intersecting, for some $s \in \mathbb{N}$, if every two edges in $S$ intersect on at most $s$ vertices.

The proof of Lemma 27 relies on the following two lemmas.

LEMMA 28. Let $k \geqslant 4$ be an integer and let $H$ be $k$-uniform hypergraph with at most $\left\lceil\frac{3}{2}(k+1)\right\rceil$ edges. Then there exists a $(k-2)$-intersecting subset $S \subseteq E(H)$ of edges such that $H \backslash S$ does not contain $T_{2 k}^{k}$.

In the proof of Lemma 27 we require Lemma 28 for a value of $k$ that is one smaller than the starting value. Thus for the case $k=4$ we would need Lemma 28 for $k=3$. Here we can only show a slightly weaker bound on the number of edges that nevertheless requires much work and whose proof can be found in the 
Appendix (since the lemma deals with hypergraphs on 7 edges, it can in principle be checked 'easily').

LEMMA 29. Let $H$ be a 3-uniform hypergraph with 7 edges. Then there exists a 1-intersecting subset $S \subseteq H$ of edges such that $H \backslash S$ does not contain a copy of $T_{6}^{3}$.

Before we prove Lemma 28 we first derive Lemma 27.

Proof of Lemma 27. Let us assume towards a contradiction that Lemma 27 is false for some $k \geqslant 4$. Then there exists a $k$-uniform hypergraph $H$ with $m(H) \leqslant$ $m_{k}\left(K_{3}^{+k}, C_{t_{k}}\right)$ and $H \rightarrow\left(K_{3}^{+k}, C_{t_{k}}\right)$ such that for every $H^{\prime}$ obtained from $H$ by removing a single vertex (and all adjacent edges) we have $H^{\prime} \nrightarrow \rightarrow\left(K_{3}^{+k}, C_{t_{k}}\right)$ (that is, $H$ is a vertex-minimal counterexample). Since we have

$$
\frac{1}{k|V(H)|} \sum_{x \in V(H)} \operatorname{deg}(x) \leqslant m(H) \leqslant m_{k}\left(K_{3}^{+k}, C_{t_{k}}\right)
$$

it follows that there exists a vertex $x \in V(H) \operatorname{such}$ that $\operatorname{deg}(x) \leqslant\left\lfloor k \cdot m_{k}\left(K_{3}^{+k}\right.\right.$, $\left.\left.C_{t_{k}}\right)\right\rfloor$. By the choice of $H$ we know that there exists a colouring of the edges in $H \backslash x$ (that is, the subgraph induced by the vertex set $V(H) \backslash\{x\})$ without a red $K_{3}^{+k}$ and a blue $C_{t_{k}}$. We now extend this colouring to the edges of $H$ incident to $x$.

Consider the link hypergraph $H_{x}$ of the vertex $x$, which is the $(k-1)$-uniform hypergraph with the edges $\{e \backslash\{x\}: e \in E(H), x \in e\}$. Let us assume that there exists a $(k-3)$-intersecting set of edges $S \subseteq E\left(H_{x}\right)$ such that $H_{x} \backslash S$ does not contain a copy of $T_{2(k-1)}^{k-1}$ (we show later that we can indeed find such a set). Let $R$ and $B$ denote the edges of $H$ obtained by adding back the vertex $x$ to the edges of $S$ and $H_{x} \backslash S$, respectively. Note that the set $R$ is $(k-2)$-intersecting since $S$ is $(k-3)$-intersecting. We claim that colouring the edges in $R$ with red and the edges in $B$ with blue gives a contradiction to the assumption $H \rightarrow\left(K_{3}^{+k}, C_{t_{k}}\right)$ :

- By the assumption on the colouring of $H \backslash x$ any red copy of $K_{3}^{+k}$ has to contain $x$ and, therefore, at least two edges from $R$. However, as every two edges of $K_{3}^{+k}$ intersect on $k-1$ vertices, the existence of such copy would contradict the fact that $R$ is $(k-2)$-intersecting.

- Similarly as in the previous case, a blue copy of $C_{t_{k}}$ necessarily contains $x$ which implies that the subgraph given by the edges in $B$ contains $T_{2 k-1}^{k}$ (since $B$ is the set of blue edges incident to $x$ and $t_{k} \geqslant 2 k$ ). Removing the vertex $x$ from every edge of such a copy gives a copy of $T_{2(k-1)}^{k-1}$ in $H_{x} \backslash S$, which is a contradiction. 
To conclude, we obtained a colouring of $H$ which contains no red $K_{3}^{+k}$ and no blue $C_{t_{k}}$, thus a contradiction with $H \rightarrow\left(K_{3}^{+k}, C_{t_{k}}\right)$.

It remains to prove that we can find an $(k-3)$-intersecting set of edges $S \subseteq H_{x}$ such that $H_{x} \backslash S$ does not contain $T_{2(k-1)}^{k-1}$. Recall that

$$
m_{k}\left(K_{3}^{+k}, C_{t_{k}}\right)=\frac{3 t_{k}-3}{2 t_{k}-k-1} .
$$

If $k=4$ then from the choice of $x$ we have

$$
\operatorname{deg}(x) \leqslant\left\lfloor 4 \cdot m_{4}\left(K_{3}^{+4}, C_{8}\right)\right\rfloor=7,
$$

and hence $H_{x}$ is a 3-uniform hypergraph with at most 7 edges. Now we can apply Lemma 29 to $H_{x}$ to obtain a set $S$ with the desired properties. Otherwise, if $k \geqslant 5$ then one can check that

$$
\operatorname{deg}(x) \leqslant\left\lfloor k \cdot m_{k}\left(K_{3}^{+k}, C_{t_{k}}\right)\right\rfloor \leqslant\left\lceil\frac{3}{2} k\right\rceil
$$

and thus $H_{x}$ is a $(k-1)$-uniform hypergraph with at most $\left\lceil\frac{3}{2} k\right\rceil$ edges. Therefore, Lemma 28 guarantees the existence of the desired set $S$. This concludes the proof.

In the next subsection we prove Lemma 28.

5.1.1. Proof of Lemma 28. We use the following two observations on the structure of tight paths.

LEMMA 30. Let $k \geqslant 3$ and let $a_{0}, a_{1}$ be two different edges of the graph $T_{2 k}^{k}$. Let $m:=\left|a_{0} \cap a_{1}\right| \geqslant 1$. Then there exist $k-m-1$ different edges $\left\{e_{1}, \ldots\right.$, $\left.e_{k-m-1}\right\} \subseteq E\left(T_{2 k}^{k}\right) \backslash\left\{a_{0}, a_{1}\right\}$ such that for each $i \in\{1, \ldots, k-m-1\}$ the following holds:

(1) $\left|e_{i} \cap a_{0} \cap a_{1}\right|=m$;

(2) $\left|e_{i} \cap\left(a_{0} \backslash a_{1}\right)\right|=i$; and

(3) $\left|e_{i} \cap\left(a_{1} \backslash a_{0}\right)\right|=k-m-i$.

Moreover, for each edge $e^{\prime} \in E\left(T_{2 k}^{k}\right) \backslash\left\{a_{0}, a_{1}, e_{1}, \ldots, e_{k-m-1}\right\}$ there exists $b \in\{0$, 1\} such that:

(i) $\left|e^{\prime} \cap a_{0} \cap a_{1}\right| \leqslant m-1$;

(ii) $\left|e^{\prime} \cap\left(a_{b} \backslash a_{1-b}\right)\right|=0$; and

(iii) $\left|e^{\prime} \cap\left(a_{1-b} \backslash a_{b}\right)\right|=k-m$. 
Proof. Let $f_{1}, \ldots, f_{k+1}$ be a natural ordering of the edges of $T_{2 k}^{k}$. As $a_{0}$ and $a_{1}$ have exactly $m$ vertices in intersection it follows that there exists an index $i \in$ $\{1, \ldots, m\}$ such that $a_{0}=f_{i}$. Since the reversed ordering of the edges (that is, $\left.f_{i}^{\prime}=f_{k+1-i}\right)$ is also a natural ordering, we can assume that $a_{0}$ is 'to the left' of $a_{1}$ and thus $a_{1}=f_{i+k-m}$. It is easy to see that the set of edges $e_{k-m-j}=f_{i+j}$ (for $1 \leqslant j \leqslant k-m-1$ ) satisfies properties $1-3$. Consequently, each edge $e^{\prime} \in$ $E\left(T_{2 k}^{k}\right) \backslash\left\{a_{0}, a_{1}, e_{1}, \ldots, e_{k-m-1}\right\}$ has to correspond to an edge from either $\left\{f_{1}, \ldots\right.$, $\left.f_{i-1}\right\}$ or $\left\{f_{i+k-m+1}, \ldots, f_{k+1}\right\}$. In both of these cases it is easy to see that properties (i)-(iii) hold.

Claim 31. The largest $(k-2)$-intersecting set in $T_{k+\ell-1}^{k}$ is of size $\lceil\ell / 2\rceil$.

Proof. Let $\left\{e_{1}, \ldots, e_{\ell}\right\}$ be a natural order of the edges of $T_{k+\ell-1}^{k}$. Observe that the set

$$
\left\{e_{1}, e_{3}, \ldots, e_{2\lceil\ell / 2\rceil-1}\right\}
$$

is $(k-2)$-intersecting and has size $\lceil\ell / 2\rceil$. Any set of more than $\lceil\ell / 2\rceil$ edges contains two edges $e_{i}$ and $e_{i+1}$, for some $i \in\{1, \ldots, \ell-1\}$. Such a set cannot be $(k-2)$-intersecting as by definition $\left|e_{i} \cap e_{i+1}\right|=k-1$.

We are now ready to prove Lemma 28.

Proof of Lemma 28. If $H$ does not contain $T_{2 k}^{k}$ then $S:=\emptyset$ satisfies the required properties. Otherwise, from Claim 31 we have that $H$ contains a $(k-2)$ intersecting set $S$ of size $\lceil(k+1) / 2\rceil$. Let $H^{\prime}:=H \backslash S$ and note that $H^{\prime}$ has at most

$$
\left\lceil\frac{3}{2}(k+1)\right\rceil-\lceil(k+1) / 2\rceil=k+1
$$

edges. If $H^{\prime}$ is not isomorphic to $T_{2 k}^{k}$ then $S$ satisfies the required properties. Otherwise, let $E\left(H^{\prime}\right)=\left\{h_{1}, \ldots, h_{k+1}\right\}$ be a natural order of the edges of $H^{\prime}$ and label the vertices $V\left(H^{\prime}\right)=\left\{v_{1}, \ldots, v_{2 k}\right\}$ such that

$$
h_{i}=\left\{v_{i}, \ldots, v_{i+k-1}\right\}
$$

for all $1 \leqslant i \leqslant k+1$. Note that

$$
C_{1}:=\left\{h_{1}, h_{3}, \ldots, h_{2\lceil(k+1) / 2\rceil-1}\right\} \quad \text { and } \quad C_{2}:=\left\{h_{2}, h_{4}, \ldots, h_{2\lfloor(k+1) / 2\rfloor}\right\}
$$

are $(k-2)$-intersecting sets of size $\left|C_{1}\right|=\lceil(k+1) / 2\rceil$ and $\left|C_{2}\right|=\lfloor(k+1) / 2\rfloor$. We show that $H \backslash C_{i}$ does not contain $T_{2 k}^{k}$ for some $i \in\{1,2\}$.

Let us assume towards a contradiction that this is not the case and let $F_{1}$ and $F_{2}$ denote arbitrarily chosen copies of $T_{2 k}^{k}$ in $C_{1} \cup S$ and $C_{2} \cup S$, respectively. Since $\left|C_{1} \cup S\right| \leqslant k+2$ there can be at most one edge in $C_{1} \cup S$ which is not part of $F_{1}$. 
We first show that $h_{1} \in E\left(F_{1}\right)$ implies $h_{3} \in E\left(F_{1}\right)$. Let us assume towards the contradiction that $h_{1} \in E\left(F_{1}\right)$ and $h_{3} \notin E\left(F_{1}\right)$. Since $F_{1}$ contains all but at most one edge in $C_{1} \cup S$, this implies that a subgraph induced by edges $S \cup C_{1} \backslash\left\{h_{3}\right\}$ is isomorphic to $T_{2 k}^{k}$. Let $E\left(F_{1}\right)=\left\{f_{1}, \ldots, f_{k+1}\right\}$ be a natural order of the edges of $F_{1}$ and let $j$ be such that $f_{j}=h_{1}$. We can assume that $j \leqslant\lceil k / 2\rceil$ as otherwise we can just reverse the order of the edges. Since $j+2 \leqslant k$ for $k \geqslant 5$, the edges $f_{j+1}$ and $f_{j+2}$ are such that

$$
\left|f_{j+1} \cap f_{j+2}\right|=k-1, \quad\left|f_{j+1} \cap f_{j}\right|=k-1, \quad \text { and } \quad\left|f_{j+2} \cap f_{j}\right|=k-2 .
$$

However, no edge from $C_{1} \backslash\left\{h_{1}, h_{3}\right\}$ intersects $h_{1}$ on more than $k-4$ vertices, which implies that both $f_{j+1}$ and $f_{j+2}$ are contained in $S$. As $S$ is a $(k-2)$ intersecting set and $\left|f_{j+1} \cap f_{j+2}\right|=k-1$, this gives a contradiction. To conclude, we showed that if $h_{1} \in E\left(F_{1}\right)$ then $h_{3} \in E\left(F_{1}\right)$.

Note that the previous observation together with the fact that $F_{1}$ contains all but at most one edge in $C_{1} \cup S$ implies that either $\left\{h_{1}, h_{3}\right\} \subseteq E\left(F_{1}\right)$ or $\left\{h_{3}\right.$, $\left.h_{5}\right\} \subseteq E\left(F_{1}\right)$ (or both). We only consider the first case as the latter follows by a symmetric argument. From Lemma 30 with $a_{0}=h_{1}$ and $a_{1}=h_{3}($ and $m=k-2)$ we conclude that there exists an edge $x \in E\left(F_{1}\right)$ such that

$$
\left|x \cap h_{1} \cap h_{3}\right|=k-2, \quad\left|x \cap\left(h_{1} \backslash h_{3}\right)\right|=1 \quad \text { and } \quad\left|x \cap\left(h_{3} \backslash h_{1}\right)\right|=1 .
$$

Therefore we have $\left|x \cap h_{1}\right|=k-1$ and as $C_{1}$ is a $(k-2)$-intersecting set we have $x \in S$.

Next, let us look at $F_{2}$. As $\left|C_{2}\right|=\lfloor(k+1) / 2\rfloor$ we have $\left|S \cup C_{2}\right| \leqslant k+1$. Therefore, $E\left(F_{2}\right)=S \cup C_{2}$ and from $x \in S$ we conclude $x \in E\left(F_{2}\right)$. Using (16) we obtain

$$
\begin{aligned}
& \left|x \cap h_{2} \cap h_{4}\right|=k-3+I\left(v_{k+1}\right), \\
& \left|x \cap\left(h_{2} \backslash h_{4}\right)\right|=1+I\left(v_{2}\right), \quad \text { and } \\
& \left|x \cap\left(h_{4} \backslash h_{2}\right)\right|=I\left(v_{k+2}\right)+I\left(v_{k+3}\right),
\end{aligned}
$$

where

$$
I(v):= \begin{cases}1 & v \in x, \\ 0 & \text { otherwise. }\end{cases}
$$

Observe that by (16) we also have $V(x) \subseteq V\left(h_{1}\right) \cup V\left(h_{3}\right)$ and since $v_{k+3} \notin$ $V\left(h_{1}\right) \cup V\left(h_{3}\right)$ we get $I\left(v_{k+3}\right)=0$. Moreover, as $\left|x \cup\left(h_{3} \backslash h_{1}\right)\right|=1$ and $h_{3} \backslash h_{1}=$ $\left\{v_{k+1}, v_{k+2}\right\}$ we conclude

$$
I\left(v_{k+1}\right)=1-I\left(v_{k+2}\right) .
$$

These observations imply that there are only two possibilities: 
- $\left|x \cap h_{2} \cap h_{4}\right|=k-2$ and $\left|x \cap\left(h_{4} \backslash h_{2}\right)\right|=0$; or

- $\left|x \cap h_{2} \cap h_{4}\right|=k-3,\left|x \cap\left(h_{4} \backslash h_{2}\right)\right|=1$ and $\left|x \cap\left(h_{2} \backslash h_{4}\right)\right| \geqslant 1$.

By applying Lemma 30 with $a_{0}=h_{2}$ and $a_{1}=h_{4}$ (and $m=k-2$ ) one can see that no edge from $F_{2} \backslash\left\{h_{2}, h_{4}\right\}$ can satisfy either of the two possibilities. This gives a contradiction with the assumption that both $H \backslash C_{1}$ and $H \backslash C_{2}$ contain $T_{2 k}^{k}$, which concludes the proof of the lemma.

5.2. Property (iii). In this subsection we prove that property (iii) from Theorem 8 (the asymmetric-balancedness) holds for $F_{1}=K_{3}^{+k}$ and $F_{2}=C_{t_{k}}$. For convenience of the reader, we state it as a lemma.

LEMMA 32. The family $\mathcal{C}^{*}=\mathcal{F}^{*}\left(K_{3}^{+k}, C_{t_{k}}\right)$ is asymmetric-balanced for every $k \geqslant 4$ (see Definition 7).

Consider some graph $C^{*} \in \mathcal{C}^{*}$. Recall that by the definition of $\mathcal{F}^{*}\left(K_{3}^{+k}, C_{t_{k}}\right)$ there exists a subgraph $C_{t_{k}}^{*} \subseteq C^{*}$ isomorphic to $C_{t_{k}}$ and an ordering $e_{0}, \ldots, e_{t_{k}-1}$ of the edges of $C_{t_{k}}^{*}$ such that for each $1 \leqslant i \leqslant t_{k}-1$ there exists a subgraph $F^{i} \subseteq C^{*}$ which contains an edge $e_{i}$ and is isomorphic to $K_{3}^{+k}$. Recall that we refer to the edge $e_{0}$ as an attachment edge. For simplicity, we assume that the vertices of $C_{t_{k}}^{*}$ are labelled with numbers $\left\{0, \ldots, t_{k}-1\right\}$ such that $e_{i}=\{i, i+1, \ldots, k-1+i\}$ (where all additions are modulo $t_{k}$ ) for $0 \leqslant i \leqslant t_{k}-1$. Moreover, let $v_{i}$ denote the vertex such that $e_{i} \cup\left\{v_{i}\right\}=V\left(F^{i}\right)$ (for $1 \leqslant i \leqslant t_{k}-1$ ). Notice that $v_{i}$ s need not be distinct.

We make a few observations that will lead us to a crucial calculation.

Claim 33. Let $I \subseteq\left\{i: v_{i} \notin V\left(C_{t_{k}}^{*}\right)\right\}$ and $S=\left\{v_{i}: i \in I\right\}$. Then the number of edges from $C^{*}$ incident to $S$ is at least $|I|+|S|$.

Proof. Set $s:=|S|$ and let $u_{1}, \ldots, u_{s}$ be the vertices of $S$. Since every edge in $C^{*}$ contains at most one vertex in $S$, we are interested in estimating $\sum_{j=1}^{s} \operatorname{deg}_{C^{*}}\left(u_{j}\right)$. For every vertex $u_{j}$ we denote by $W(j)$ the set of the indices $i \in I$ such that $F^{i}$ contains $u_{j}$ and we set $w(j):=|W(j)|$. Observe that the sets $W(j)$ partition $I$.

Consider two distinct indices $i_{1}$ and $i_{2}$ from $W(j)$, for some $1 \leqslant j \leqslant s$. First, note that $F^{i_{1}}$ and $F^{i_{2}}$ have at most one edge in intersection. Otherwise we would have $V\left(F^{i_{1}}\right)=V\left(F^{i_{2}}\right)$ which implies $e_{i_{1}}=e_{i_{2}}$, contradicting $i_{1} \neq i_{2}$. Moreover, if $F^{i_{1}}$ and $F^{i_{2}}$ share an edge then, because any two edges of $K_{3}^{+k}$ have intersection of size $k-1$, we necessarily have that $e_{i_{1}}$ and $e_{i_{2}}$ are consecutive edges, that is, $\left|i_{1}-i_{2}\right|=1$ (note that $0 \notin W(j)$ ). 
Let $W(j)=\left\{j_{1}, \ldots, j_{q}\right\}$ where $j_{i} \leqslant j_{i+1}$. Using the previous observation we estimate $\operatorname{deg}_{C^{*}}\left(u_{j}\right) \geqslant w(j)+1$ by counting the contribution of each $F^{j_{i}}$ in the increasing order. In particular, $F^{j_{1}}$ contributes two edges and every further $F^{j_{i}}$ at least one new edge. This is clearly true for $i<q$. If $F^{j_{q}}$ does not contribute any new edge then from the previous observation we conclude that $e_{j_{q-1}}, e_{j_{q}}$ and $e_{j_{1}}$ are consecutive edges. This, however, cannot be because $0 \notin W(j)$. To conclude, we obtain

$$
\sum_{j=1}^{s} \operatorname{deg}_{C^{*}}\left(u_{j}\right) \geqslant s+\sum_{j=1}^{s} w(j)=|S|+|I| .
$$

Claim 34. Suppose an edge e $\in C^{*}$ belongs to $F^{i}, F^{j}$ and $F^{\ell}$, for some $1 \leqslant i<$ $j<\ell \leqslant t_{k}-1$. Then the edges $e_{i}, e_{j}$ and $e_{\ell}$ are consecutive (that is, $j=i+1$ and $\ell=i+2$ ) and

$e=\{i, i+2, \ldots, i+k\} \quad$ or $\quad e=\{i+1, \ldots, i+k-1, i+k+1\} \quad$ or $\quad e=e_{i+1}$.

In particular, we have $e \subseteq V\left(C_{t_{k}}^{*}\right)$.

Proof. Since any two edges of $K_{3}^{+k}$ have intersection of size $k-1$ it follows that $e_{i}, e_{j}$ and $e_{\ell}$ must pairwise intersect in at least $k-2$ vertices. This is only possible if these edges are three consecutive edges on the cycle $C_{t_{k}}^{*}$.

If $e \in E\left(C_{t_{k}}^{*}\right)$ then from $\left|e \cap e_{i+j}\right| \geqslant k-1$ for $j \in\{0,1,2\}$ we conclude $e=e_{i+1}$. Next, suppose that $e \notin E\left(C_{t_{k}}^{*}\right)$. Note that then $e$ has to contain $e_{i} \cap e_{i+2}$. This can be seen as follows: from $\left|e \cap e_{i}\right|=\left|e \cap e_{i+2}\right|=k-1$ we have $\mid e \cap\left(e_{i} \cap\right.$ $\left.e_{i+2}\right) \mid \geqslant k-3$ (that is, $e$ can 'miss' at most one vertex from $e_{i} \cap e_{i+2}$ ). However, if $\left|e \cap\left(e_{i} \cap e_{i+2}\right)\right|=k-3$ then necessarily $e_{i} \backslash e_{i+2} \subset e$ and $e_{i+2} \backslash e_{i} \subset e$ which implies $|e| \geqslant k+1$, thus a contradiction. Consequently, exactly one vertex from $\{i, i+1\}$ and one vertex from $\{i+k, i+k+1\}$ belongs to $e$. Moreover, from $\left|e \cap e_{i+1}\right|=k-1$ we deduce $\{i, i+k+1\} \not \subset e$ and from $e \notin E\left(C_{t_{k}}^{*}\right)$ we have $\{i+1, i+k\} \not \subset e$ (as otherwise $e=e_{i+1}$ ). This leads to the two remaining shapes of $e$.

Given $j \in\left[t_{k}-1\right]$ such that $V\left(F^{j}\right) \subseteq V\left(C_{t_{k}}^{*}\right)$, we denote by $E^{j}$ the set of the noncycle edges from $F^{j}$, that is,

$$
E^{j}:=\left\{e \in E\left(F^{j}\right): e \notin E\left(C_{t_{k}}^{*}\right) \text { and } e \subseteq V\left(C_{t_{k}}^{*}\right)\right\} .
$$

ClAIM 35. If an edge $e \notin C_{t_{k}}^{*}$ belongs to $F^{i}, F^{i+1}$ and $F^{i+2}$ then there is an edge $e^{\prime}$ in $E^{i} \cup E^{i+1} \cup E^{i+2}$ such that $\{i, i+k+1\} \subseteq e^{\prime}$ and $e^{\prime}$ belongs to at most two hypergraphs $F^{j}$. 
Proof. From Claim 34 we know that either $e=\{i, i+2, \ldots, i+k\}, e=\{i+1, \ldots$, $i+k-1, i+k+1\}$ or $e=e_{i+1}$. Assume first that $e=\{i+1, \ldots, i+k-1, i+k+1\}$. Then the edge $e^{\prime} \in E^{i} \backslash\{e\}$ must contain $i$ and $i+k+1$. If $e=\{i, i+2, \ldots, i+k\}$ then the edge $e^{\prime} \in E^{i+2} \backslash\{e\}$ must contain $i$ and $i+k+1$. Again from Claim 34 we conclude that if $e^{\prime}$ is contained in three different $F^{j}$ s then it cannot contain both $i$ and $i+k+1$, thus a contradiction. Therefore, $e^{\prime}$ is the desired edge.

Claim 36. Let $F^{i}$ be such that $V\left(F^{i}\right) \subseteq V\left(C_{t_{k}}^{*}\right)$. If $\left|E^{i}\right|=1$ then either $e_{i-1}$ or $e_{i+1} \in E\left(F^{i}\right)$.

Proof. This follows immediately from the fact that any two edges of $K_{3}^{+k}$ have intersection of size $k-1$.

For a given subset $X \subseteq\left\{k, \ldots, t_{k}-1\right\}$ we define $E_{X}$ to be the set of all edges $e$ of $C_{t_{k}}^{*}$ such that either the leftmost vertex of $e$ lies in $X$ or $\min X \in e$, that is,

$$
E_{X}=\left\{e_{i}: i \in X\right\} \cup\left\{e \in E\left(C_{t_{k}}^{*}\right): \min X \in e\right\} .
$$

Claim 37. Let $X \subseteq\left\{k, \ldots, t_{k}-1\right\}$, and let

$$
I=\left\{i: e_{i} \in E_{X} \text { and } v_{i} \notin V\left(C_{t_{k}}^{*}\right)\right\}
$$

and $I^{\prime} \subseteq$ I. If $|X|>3$ then the number of edges in $C^{*}$ that intersect $X$ and are either contained in $V\left(C_{t_{k}}^{*}\right)$ or intersect $\left\{v_{i}: i \in I^{\prime}\right\}$ is at least

$$
(|X|+k-1)+\frac{|X|+k-1-|I|}{2}+\frac{\left|I^{\prime}\right|}{2} .
$$

Proof. We write $X=\left\{x_{1}<\cdots<x_{m}\right\}(m \geqslant 4)$. There are exactly $m$ edges in $C_{t_{k}}^{*}$ whose leftmost vertex lies in $X$ (namely $e_{x_{1}}, \ldots, e_{x_{m}}$ ). Moreover, there are further $k-1$ edges $e \in E\left(C_{t_{k}}^{*}\right)$ with $x_{1} \in e$ (note that from $x_{1} \geqslant k$ we have that these edges are different from $e_{x_{i}}$ ) and therefore $\left|E_{X}\right|=|X|+k-1$. Clearly, by the definition we have that each $e \in E_{X}$ intersects $X$ and $e \subseteq V\left(C_{t_{k}}^{*}\right)$ thus this establishes the first group of summands in (17).

Next, we estimate those edges which contain a vertex from $\left\{v_{i}: i \in I^{\prime}\right\}$ and $X$. Since $v_{i} \notin V\left(C_{t_{k}}^{*}\right)$ and $e_{i} \cap X \neq \emptyset$ for $i \in I^{\prime}$ (this follows from $e_{i} \in E_{X}$ ), we deduce that at least one of the edges from $E\left(F^{i}\right) \backslash\left\{e_{i}\right\}$ must intersect $X$ and contain $v_{i}$ (recall that in $K_{3}^{+k}$ any pair of vertices is connected by an edge). Moreover, from Claim 34 we have that every such edge $e$ belongs to at most two $F^{i}$ s, as otherwise we have $v_{i} \in e \subseteq V\left(C_{t_{k}}^{*}\right)$ which contradicts $v_{i} \notin V\left(C_{t_{k}}^{*}\right)$. This shows that the number of edges incident both to $X$ and $\left\{v_{i}\right\}_{i \in I^{\prime}}$ is at least $\left|I^{\prime}\right| / 2$, which establishes the third summand in (17). 
It remains to show that there are at least

$$
\frac{|X|+k-1-|I|}{2}
$$

edges $e \in E\left(C^{*}\right) \backslash E_{X}$ such that $e \cap X \neq \emptyset$ and $e \subseteq V\left(C_{t_{k}}^{*}\right)$. Let

$$
\begin{aligned}
E & :=\left\{e_{i} \in E_{X}: v_{i} \in V\left(C_{t_{k}}^{*}\right)\right\}, \\
E_{1} & :=\left\{e_{i} \in E_{X}: v_{i} \in V\left(C_{t_{k}}^{*}\right) \text { and } \exists e \in E^{i} \text { such that } e \cap X=\emptyset\right\}, \\
E_{2} & :=\left\{e_{i} \in E_{X}: v_{i} \in V\left(C_{t_{k}}^{*}\right) \text { and } \forall e \in E^{i} \text { we have } e \cap X \neq \emptyset\right\} .
\end{aligned}
$$

Observe that $E=E_{1} \dot{\cup} E_{2}$ holds. Furthermore set

$$
\begin{aligned}
E^{\prime} & :=\bigcup_{e_{j} \in E_{2}} E^{j}, \\
\hat{E} & =\left\{e \in E\left(C_{t_{k}}^{*}\right) \backslash E_{X}: e \cap X \neq \emptyset\right\} .
\end{aligned}
$$

Note that $E^{\prime} \cap \hat{E}=\emptyset$ (recall that $E^{j} \cap E\left(C_{t_{k}}^{*}\right)=\emptyset$ by the definition). Moreover, for every edge $e \in E^{\prime} \cup \hat{E}$ we have $e \cap X \neq \emptyset$ and $e \notin E_{X}$. Here the first property follows directly from the definition of the sets $E^{\prime}$ and $\hat{E}$, whereas the second one follows from the definition of the sets $E^{j}$ and $\hat{E} \cap E_{X}=\emptyset$.

Moreover, for every $e_{i} \in E$ we have $i \notin I$, thus

$$
\left|E_{1}\right|+\left|E_{2}\right|=|E|=\left|E_{X}\right|-|I|=|X|+k-1-|I| .
$$

We estimate the sizes of $E^{\prime}$ and $\hat{E}$ separately.

- $\left|E^{\prime}\right| \geqslant\left|E_{2}\right| / 2$

Let $S_{3}$ be the set of those edges from $E^{\prime}$ that belong to at least three sets $E^{j}$ for some $j$ such that $e_{j} \in E_{2}$. From Claims 34 and 35 we deduce that if an edge $e \in E^{\prime}$ belongs to some $F^{i_{1}}, F^{i_{2}}$ and $F^{i_{3}}$ then there is another edge $e^{\prime} \in E^{\prime}$ which also belongs to $E^{i_{1}} \cup E^{i_{2}} \cup E^{i_{3}}$ but lies in at most two copies of some $F^{i}$. Therefore, the number of $E^{j} \mathrm{~s}$ (where $j \in\left\{i: e_{i} \in E_{2}\right\}$ ) such that $E^{j} \subseteq S_{3}$ is at most $2\left|S_{3}\right|$ (as otherwise there is an edge $e \in E^{i_{1}} \cup E^{i_{2}} \cup E^{i+3} \subseteq S_{3}$ which is contained in only two different $F^{i}$, s, which is a contradiction). It follows that $\left|E^{\prime}\right| \geqslant\left(\left|E_{2}\right|-2\left|S_{3}\right|\right) / 2+\left|S_{3}\right|=\left|E_{2}\right| / 2$ holds.

- $|\hat{E}| \geqslant\left|E_{1}\right| / 2$

By the definition of $E_{1}$, any edge $e_{i} \in E_{1}$ intersects $X$ in exactly one vertex. In what follows we construct a function $f: E_{1} \rightarrow \hat{E}$ where every edge of $\hat{E}$ has at most two preimages. This will then imply $|\hat{E}| \geqslant\left|E_{1}\right| / 2$. 
Given an edge $e \in E_{1}$ let $x_{i}$ be the only vertex from $X$ that is contained in $e$. If $e \neq e_{x_{1}-k+1}$ then $x_{i}$ is not the rightmost vertex in the edge $e$. If in addition $i<m$ then we define another edge $f(e)$ as follows: let $f(e) \in E\left(C_{t_{k}}^{*}\right)$ be an edge which contains $x_{i+1}$, where $x_{i+1}$ is preceded by as many vertices as there are vertices in $e$ that come after $x_{i}$. Clearly, $f(e) \notin E_{X}$ and $f(e) \cap X \neq \emptyset$. We observe that such edges $f(e)$ are all distinct and in particular $f: E_{1} \backslash\left\{e_{x_{1}-k+1}\right.$, $\left.e_{x_{m}}\right\} \rightarrow \hat{E}$ is injective. Thus, $|\hat{E}| \geqslant\left|E_{1} \backslash\left\{e_{x_{1}-k+1}, e_{x_{m}}\right\}\right|$ and if $\mid E_{1} \backslash\left\{e_{x_{1}-k+1}\right.$, $\left.e_{x_{m}}\right\}|\geqslant| E_{1} \mid / 2$ then we are done.

Therefore, it remains to consider the cases when $e_{x_{1}-k+1} \in E_{1}$ or $e_{x_{m}} \in E_{1}$ holds, where we define some more edges of the 'type' $f(e)$. This amounts to a somewhat tedious case distinction. If $e_{x_{1}-k+1} \in E_{1}$ then we first additionally assume that either $\left(x_{1}+1\right) \notin X$ or $\left(x_{1}+2\right) \notin X$ is the case. In particular, if $\left(x_{1}+1\right) \notin X$ then we define $f\left(e_{x_{1}-k+1}\right):=e_{x_{2}-1}$ and if $\left(x_{1}+2\right) \notin X$ (and $\left(x_{1}+1\right) \in X$ is) then we set $f\left(e_{x_{1}-k+1}\right):=e_{x_{3}-1}$. Similarly, if $e_{x_{m}} \in E_{1}$ and $\left(x_{m}-1\right) \notin X$ then we set $f\left(e_{x_{m}}\right):=e_{x_{m}-1}$ and if $\left(x_{m}-2\right) \notin X$ (and $\left(x_{m}-1\right) \in X$ is) we set $f\left(e_{x_{m}}\right):=e_{x_{m-1}-1}$. Notice that $f\left(e_{x_{1}-k+1}\right) \neq f\left(e_{x_{m}}\right)$ unless $m=4$, $x_{2}=x_{1}+1, x_{4}=x_{3}+1$ and $\left(x_{1}+2\right) \notin X$ (recall that by assumption $m \geqslant$ $4)$. In any case, it is easy to check that at most two $f(e)$ s are pairwise equal. Therefore, $|\hat{E}| \geqslant\left|E_{1}\right| / 2$.

Finally we treat the case where at least one of the remaining options holds: $e_{x_{1}-k+1} \in E_{1},\left(x_{1}+1\right),\left(x_{1}+2\right) \in X$ or $e_{x_{m}} \in E_{1}$ and $\left(x_{m}-1\right),\left(x_{m}-2\right) \in X$. If $e_{x_{1}-k+1} \in E_{1}$ and $\left(x_{1}+1\right),\left(x_{1}+2\right) \in X$, it follows from Claim 35 and $e_{x_{1}-k} \notin E_{X}$, that none of the edges from $E^{x_{1}-k+1}$ lies in three copies $F^{i}$ (where all $i \in\left\{j: e_{j} \in E\right\}$ ) - otherwise these have to be $F^{x_{1}-k+1}, F^{x_{1}-k+2}$ and $F^{x_{1}-k+3}$. But then with Claim 34 it follows that $F^{x_{1}-k+1}$ intersects $X$ in two vertices, which implies $e_{x_{1}-k+1} \in E_{2}$ (a contradiction). Moreover, it holds by definition that $\left|E^{x_{1}-k+1}\right| \in\{1,2\}$. And it follows further from Claim 36 that if $\left|E^{x_{1}-k+1}\right|=1$ then either $e_{x_{1}-k}$ or $e_{x_{1}-k+2}$ lies in $F^{x_{1}-k+1}$, which again implies that $e_{x_{1}-k+1} \in E_{2}$. Thus, we have $\left|E^{x_{1}-k+1}\right|=2$. Next we delete one edge from $E^{x_{1}-k+1}$ which does not intersect $X$ (and still denote the set by $\left.E^{x_{1}-k+1}\right)$. Similarly, if $e_{x_{m}} \in E_{1}$ and $\left(x_{m}-1\right),\left(x_{m}-2\right) \in X$ then none of the edges from $E^{x_{m}}$ lies in three copies $F^{i}$ (where all $i \in\left\{j: e_{j} \in E\right\}$ ). Again we have $\left|E^{x_{m}}\right|=2$ and we delete from $E^{x_{m}}$ the edge that does not intersect $X$. In each of the cases, we add $e_{x_{1}-k+1}$ (respectively $e_{x_{m}}$ ) to $E_{2}$ (and remove them from $E_{1}$ ) and the edges from $E^{x_{1}-k+1}$ (respectively $E^{x_{m}}$ ) to $E^{\prime}$ (but keeping the same notation). Now, a short meditation reveals that the same argumentation as above applies to these slightly altered sets $E_{1}$ and $E_{2}$ to show $\left|E^{\prime}\right| \geqslant\left|E_{2}\right| / 2$ and $|\hat{E}| \geqslant\left|E_{1}\right| / 2$. Indeed, the inequality $\left|E^{\prime}\right| \geqslant\left|E_{2}\right| / 2$ holds since edges $e_{x_{1}-k+1}$ and $e_{x_{m}}$ belong to at most two copies of $F^{j}$ (with $j \in\left\{i: e_{i} \in E_{2}\right\}$ ) and thus the 
estimate $\left|E^{\prime}\right| \geqslant\left(\left|E_{2}\right|-2\left|S_{3}\right|\right) / 2+\left|S_{3}\right|=\left|E_{2}\right| / 2$ remains valid. Whereas the inequality $|\hat{E}| \geqslant\left|E_{1}\right| / 2$ holds since we exclude $e_{x_{1}-k+1}$ and/or $e_{x_{m}}$ from $E_{1}$ that leads to a simpler function $f: E_{1} \rightarrow \hat{E}$, whose property that each element has at most two preimages remains valid.

The claim now follows from (19) and previously obtained bounds.

Now we are ready to prove Lemma 32.

Proof of Lemma 32. Consider some $C^{*} \in \mathcal{C}^{*}$ and suppose $H \subseteq C^{*}$ contains the attachment edge $e_{0}$ and $v(H)<v\left(C^{*}\right)$. Let

$$
S_{C}:=V\left(C^{*}\right) \backslash\left(V\left(C_{t_{k}}^{*}\right) \cup V(H)\right) \quad \text { and } \quad S_{H}:=V(H) \backslash V\left(C_{t_{k}}^{*}\right)
$$

be the set of 'outside' vertices of $C^{*}$ partitioned into those which are contained in $H$ and the rest. Furthermore, set

$$
I:=\left\{i: v_{i} \notin V\left(C_{t_{k}}^{*}\right) \cup V(H)\right\} .
$$

Clearly, $S_{C}=\left\{v_{i}: i \in I\right\}$. Furthermore, let $X \subset V\left(C_{t_{k}}^{*}\right)$ be such that $S_{C} \dot{\cup} X=$ $V\left(C^{*}\right) \backslash V(H)$, that is,

$$
X:=V\left(C_{t_{k}}^{*}\right) \backslash V(H) .
$$

Recall that $E_{X}$ consists of those edges $e$ of $C_{t_{k}}^{*}$ such that either their leftmost vertex of $e$ lies in $X$ or $\min X \in e$. We aim to lower bound $e\left(C^{*}\right)-e(H)$ in terms of $\left|S_{C}\right|$ and $|X|$. Since $v\left(C^{*}\right)-v(H)=\left|S_{C}\right|+|X|$ this enables us to bound the ratio $\left(e\left(C^{*}\right)-e(H)\right) /\left(v\left(C^{*}\right)-v(H)\right)$ from below.

Assume first that $|X| \geqslant 4$. Let us denote by $E_{1} \subseteq E\left(C^{*}\right)$ the subset of all edges which intersect $S_{C}$. From Claim 33 we obtain

$$
\left|E_{1}\right| \geqslant|I|+\left|S_{C}\right| \text {. }
$$

Note that from the definition of $S_{C}$ we have that no edge $e \in E(H)$ intersects $S_{C}$, and thus it holds $E_{1} \subseteq E\left(C^{*}\right) \backslash E(H)$. Let us denote

$$
J:=\left\{j: v_{j} \notin V\left(C_{t_{k}}^{*}\right) \text { and } e_{j} \in E_{X}\right\} .
$$

From Claim 37 we know that the number of edges $e \in E\left(C^{*}\right) \backslash E(H)$ that intersect $X$ and are either contained in $V\left(C_{t_{k}}^{*}\right)$ or intersect $\left\{v_{i}: i \in J \backslash I\right\} \subseteq S_{H}$ is at least

$$
|X|+k-1+\frac{|X|+k-1-|J|}{2}+\frac{|J \backslash I|}{2} .
$$

Let us denote such set of edges with $E_{2}$. Note that in order to apply Claim 37 we also need that $X \cap\{0, \ldots, k-1\}=\emptyset$, which follows from the assumption 
$e_{0} \in E(H)$. We have $E_{2} \cap E(H)=\emptyset$ as each edge in $E_{2}$ intersects $X$ which is a set disjoint from $V(H)$. Furthermore, we claim that $E_{2}$ is disjoint from $E_{1}$. Every edge in $C^{*}$ intersects the set of 'outside' vertices $V\left(C^{*}\right) \backslash V\left(C_{t_{k}}^{*}\right)$ on at most one vertex. Let $e^{\prime} \in E_{2}$ be an arbitrary edge from $E_{2}$. If $e^{\prime}$ is contained in $V\left(C_{t_{k}}^{*}\right)$ then $e^{\prime} \notin E_{1}$. Otherwise, we know that $e^{\prime}$ intersects $\left\{v_{i}: i \in J \backslash I\right\} \subseteq S_{H}$. As $S_{H}$ is disjoint from $S_{C}$ we conclude $e^{\prime} \notin E_{1}$ and thus $E_{1} \cap E_{2}=\emptyset$. Having this observation, together with the fact $E_{1} \cup E_{2} \subseteq E\left(C^{*}\right) \backslash E(H)$, we bound $e\left(C^{*}\right)-$ $\boldsymbol{e}(H)$ by using (20) and (21) as follows,

$$
\begin{aligned}
& e\left(C^{*}\right)-e(H) \geqslant\left|E_{1}\right|+\left|E_{2}\right| \geqslant|I|+\left|S_{C}\right|+|X|+k-1 \\
& \quad+\frac{|X|+k-1-|J|}{2}+\frac{|J \backslash I|}{2} .
\end{aligned}
$$

On the other hand we have $v\left(C^{*}\right)-v(H)=\left|S_{C}\right|+|X|$. Therefore we obtain

$$
\begin{aligned}
\frac{e\left(C^{*}\right)-e(H)}{v\left(C^{*}\right)-v(H)} & \geqslant \frac{|I|+\left|S_{C}\right|+|X|+k-1+\frac{|X|+k-1-|J|}{2}+\frac{|J \backslash I|}{2}}{\left|S_{C}\right|+|X|} \\
& =1+\frac{2|I|+3(k-1)+|X|-|J|+|J \backslash I|}{2\left(\left|S_{C}\right|+|X|\right)} \\
& \geqslant 1+\frac{|I|+3(k-1)+|X|-|J|+|J|}{2\left(\left|S_{C}\right|+|X|\right)} \\
& \left.\geqslant \geqslant|I| S_{C} \mid\right) \\
& =\frac{3}{2}+\frac{3(k-1)}{2\left(\left|S_{C}\right|+|X|+3(k-1)\right.} \\
&
\end{aligned}
$$

By comparing this with

$$
m_{k}\left(K_{3}^{+k}, C_{t_{k}}\right)=\frac{3 t_{k}-3}{2 t_{k}-k-1}=\frac{3}{2}+\frac{3(k-1)}{2\left(2 t_{k}-k-1\right)},
$$

we see that

$$
\frac{e\left(C^{*}\right)-e(H)}{v\left(C^{*}\right)-v(H)} \geqslant \frac{3}{2}+\frac{3(k-1)}{2\left(\left|S_{C}\right|+|X|\right)} \geqslant \frac{3}{2}+\frac{3(k-1)}{2\left(2 t_{k}-k-1\right)}=m_{k}\left(K_{3}^{+k}, C_{t_{k}}\right) .
$$

The second inequality follows from $|X|=\left|V\left(C_{t_{k}}^{*}\right) \backslash V(H)\right| \leqslant t_{k}-k$ (since $H$ contains $\left.e_{0}\right)$ and $\left|S_{C}\right|=\left|V\left(C^{*}\right) \backslash\left(V\left(C_{t_{k}}^{*}\right) \cup V(H)\right)\right| \leqslant\left|V\left(C^{*}\right) \backslash V\left(C_{t_{k}}^{*}\right)\right| \leqslant t_{k}-1$. Therefore, the equality is possible only if $|X|=t_{k}-k$ and $\left|S_{C}\right|=t_{k}-1$, in which case $C^{*}$ is generic and $H$ consists only of the attachment edge.

It remains to consider the case where $|X| \in\{0,1,2,3\}$. Suppose $X=\emptyset$. Then $v\left(C^{*}\right)-v(H)=\left|S_{C}\right| \geqslant 1$. From Claim 33 we have that there are at least 
$|I|+\left|S_{C}\right| \geqslant 2\left|S_{C}\right|$ edges $e \in C^{*}$ which intersect $S_{C}$, and by the definition none of these edges belongs to $H$. Therefore $e\left(C^{*}\right)-e(H) \geqslant 2\left|S_{C}\right|$ and we get

$$
\frac{e\left(C^{*}\right)-e(H)}{v\left(C^{*}\right)-v(H)} \geqslant 2^{t_{k} \geqslant 2 k} m_{k}\left(K_{3}^{+k}, C_{t_{k}}\right) .
$$

Finally, suppose $1 \leqslant|X| \leqslant 3$. There are at least $|X|+k-1$ edges in $E\left(C_{t_{k}}^{*}\right) \backslash E(H)$ which intersect $X$. In particular, those are $e_{x}$ for each $x \in X$ and $k-1$ edges preceding $e_{x_{1}}$ where $x_{1}=\min X$ (since $x_{1} \geqslant k$ there is no double counting). Moreover, by the same argument as in the previous case we have at least $|I|+\left|S_{C}\right| \geqslant 2\left|S_{C}\right|$ edges which intersect $S_{C}$. This gives

$$
\begin{aligned}
\frac{e\left(C^{*}\right)-e(H)}{v\left(C^{*}\right)-v(H)} & \geqslant \frac{|X|+k-1+2\left|S_{C}\right|}{|X|+\left|S_{C}\right|} \\
& \geqslant 2+\frac{k-1-|X|}{|X|+\left|S_{C}\right|} \geqslant 2^{k \geqslant 4} 2^{t_{k} \geqslant 2 k} m_{k}\left(K_{3}^{+k}, C_{t_{k}}\right),
\end{aligned}
$$

as required.

\section{Concluding remarks}

The 1-statement in Theorem 5 requires $F_{1}$ be strictly balanced with respect to $m_{k}\left(\cdot, F_{2}\right)$. We use this condition in Lemmas 12 and 13 to bound the expected number of pairs of distinct copies of $F_{1}$ that share at least one edge and to apply Janson's inequality. This is exploited subsequently in the proof of Theorem 5 in the probability estimate (8) via (6). At the expense of an additional log-factor we can drop the condition on the strict balancedness and prove the following.

THEOREM 38. Let $r \geqslant 2$ and $F_{1}, \ldots, F_{r}$ be $k$-uniform hypergraphs such that $m_{k}\left(F_{1}\right) \geqslant m_{k}\left(F_{2}\right) \geqslant \cdots \geqslant m_{k}\left(F_{r}\right)>0$. Then there exists a constant $C>0$ such that for $p \geqslant C n^{-1 / m_{k}\left(F_{1}, F_{2}\right)} \log n$ we have

$$
\lim _{n \rightarrow \infty} \operatorname{Pr}\left[H^{k}(n, p) \rightarrow\left(F_{1}, \ldots, F_{r}\right)\right]=1
$$

The proof is a slight modification of the proof of Theorem 5 and we briefly sketch the argument. We exploit $H^{k}(n, p) \not \rightarrow\left(F_{1}, \ldots, F_{r}\right)$ by applying the container theorem, Theorem 15, as before with $F=F_{i}$ for $i \geqslant 2$ and with $H_{i}$ being the edges of $H^{k}(n, p)$ coloured $i$ without a copy of $F_{i}$. The tuples $T^{i}$ consist then of sets of sizes at most $\ell n^{k-1 / m_{k}\left(F_{i}\right)}$ each. Thus, a colouring of the edges of $H \sim H^{k}(n, p)$ which certifies $H \not \rightarrow\left(F_{1}, \ldots, F_{r}\right)$ allows us to place the $i$-coloured edges $H_{i}$, for $i \geqslant 2$, into some container $C_{i}$ with fewer than $\alpha n^{v\left(F_{i}\right)}$ 
copies of $F_{i}$. Thus, Lemma 11 yields, that $H^{\prime}:=K_{n}^{(k)} \backslash \bigcup_{i \geqslant 2} E\left(C_{i}\right)$ contains at least $\alpha n^{v\left(F_{1}\right)}$ copies of $F_{1}$ for some absolute $\alpha>0$. Therefore, all edges in $H^{\prime} \cap H$ are coloured in colour 1 and since $H_{1}=H^{\prime} \cap H$, none of the copies of $F_{1}$ from $H^{\prime}$ is in $H_{1}$. But then Janson's inequality, viz. Lemma 13, yields the probability of at most $e^{-\beta C n^{k-1 / m_{k}\left(F_{2}\right)} \log n}$ that none of the copies of $F_{1}$ in $H_{1}$ is present in $H$. More precisely, let $F_{1}^{\prime}$ be the subgraph of $F_{1}$ that is strictly balanced with respect to $m_{k}\left(\cdot, F_{2}\right)$. Then, Lemmas 12 and 13 are applicable to $F_{1}^{\prime}$ instead of $F_{1}$ and if $H_{1}$ does not contain a copy of $F_{1}^{\prime}$ then it does not contain a copy of $F_{1}$ as well. The probability for the latter event is, by Lemma 13 , at most $e^{-\beta C n^{k-1 / m_{k}\left(F_{2}\right)} \log n}$. The number of choices for $T^{i}$, s is at the same time at most

$$
\left(\begin{array}{c}
n^{k} \\
r \ell n^{k-1 / m_{k}\left(F_{2}\right)}
\end{array}\right) 2^{r^{2} \ell^{2} n^{k-1 / m_{k}\left(F_{2}\right)}}=e^{O_{r, \ell}\left(n^{k-1 / m_{k}\left(F_{2}\right)} \log n\right)} .
$$

But then the union bound over all choices of $T^{i}$ finishes the claim, if we choose $C$ large enough, since the failure probability $e^{-\beta C n^{k-1 / m_{k}\left(F_{2}\right)} \log n}$ times the number of choices for $T^{i} \mathrm{~s}$ is $o(1)$. It would be of interest to further remove the $\log n$ factor in Theorem 38.

Another research direction concerns sharp thresholds, already mentioned in the introduction. For example, in the case of a triangle and two colours, $G(n$, $p) \rightarrow\left(K_{3}\right)$ has a sharp threshold as proved by Friedgut et al. [10]. Building on the work of Friedgut et al. [8] on the sharpness of the threshold for the van der Waerden property in random subsets, Schacht and Schulenburg [27] gave a proof for sharpness of a threshold for a class of strictly balanced and nearly bipartite graphs, that is, those graphs that contain an edge whose deletion makes them bipartite. Such a class contains all odd cycles, and thus gives a shorter proof of the result in [10]. The new proof of the sharpness result uses an application of Friedgut's sharpness criterion [7] in combination with container theorems [2, 26]. It would be interesting to obtain sharpness results for some asymmetric graph Ramsey properties or even for hypergraphs.

In $[17,28]$ we proved the 0 -statement for the threshold probability for $H^{k}(n$, $p) \rightarrow(F)_{r}$, where $r \geqslant 2$ and $F$ is the complete $k$-uniform hypergraph. This 0 statement is of the form $c n^{-1 / m_{k}(F)}$ and thus it matches the corresponding bounds for the 1-statement due to Conlon and Gowers [5] and Friedgut et al. [11] up to a multiplicative constant. In [28], similar 0-statements are proved for larger classes of hypergraphs apart from complete graphs. We refer the interested reader to [28, Theorem 6.13]. However, we are far from having a complete characterization of the thresholds for $H^{k}(n, p) \rightarrow(F)_{r}$ for general $F$ and $r \geqslant 2$. It would be interesting to decide whether the connection between asymmetric and symmetric Ramsey properties (Theorem 3) occurs in the case of 3-uniform hypergraphs as well. 


\section{Acknowledgement}

Y. Person was supported by DFG grant PE 2299/1-1.

\section{Appendix A. Proof of Lemma 29}

For convenience of the reader we restate Lemma 29.

LEMMA. Let $H$ be a 3-uniform hypergraph with 7 edges. Then there exists a 1intersecting subset $S \subseteq H$ of edges such that $H \backslash S$ does not contain a copy of $T_{6}^{3}$.

Recall that $T_{3}^{6}$ is a 3-uniform tight path with 6 vertices. First we show a simple claim, which is used later in the proof.

Claim 39. Let $H$ be a 3-uniform hypergraph which does not contain a 1intersecting set of size three and let $x, y \in E(H)$ be two disjoint edges of $H$. Then each edge $e \in E(H) \backslash\{x, y\}$ has to intersect exactly one of the edges from $\{x, y\}$ and on exactly two vertices.

Proof. Let $e$ be an arbitrary edge from $E(H) \backslash\{x, y\}$. If $e$ intersects both $x$ and $y$ on at most one vertex then set $\{x, y, e\}$ is a 1 -intersecting set of size three, which is a contradiction with the assumption of the lemma. On the other hand, if $e$ intersects both $x$ and $y$ on two vertices, then $x$ and $y$ cannot be disjoint.

Next, we show that Lemma 29 holds if $H$, additionally, does not contain a 1-intersecting set of size three.

LEMMA 40. Let $H$ be a 3-uniform hypergraph with 7 edges and no 1-intersecting set of size three. There is a 1-intersecting subset $S \subseteq E(H)$ such that $H \backslash S$ does not contain a copy of $T_{6}^{3}$.

Proof. In order to obtain a contradiction, suppose that for every 1-intersecting set $S \subseteq E(H)$ the graph $H \backslash S$ contains a $T_{6}^{3}$-copy. We show that this assumption gives us enough information to deduce important information about the structure of $H$. Let $a$ and $b$ be two edges of an arbitrary $T_{6}^{3}$-copy of $H$ such that $a \cap b=\emptyset$. The set of edges $\{a, b\}$ is a 1 -intersecting set and thus $H \backslash\{a, b\}$ contains a $T_{6}^{3}$ copy. Pick an arbitrary such copy and denote it by $F$. Let $E(F)=\left\{e_{1}, e_{2}, e_{3}, e_{4}\right\}$ be a natural order of edges of $F$ and let us denote the only remaining edge from $E(H) \backslash\left\{a, b, e_{1}, e_{2}, e_{3}, e_{4}\right\}$ by $e^{*}$. By applying Claim 39 to $a$ and $b$ we know that edges $e_{1}, e_{4}$ and $e^{*}$ have to intersect $a$ or $b$ on exactly two vertices. Note that $e_{1}$ 
and $e_{4}$ must not intersect the same edge on two vertices as then they would not be disjoint. Let us assume without loss of generality that

$$
\left|e_{1} \cap a\right|=2, \quad\left|e^{*} \cap a\right|=2, \quad \text { and } \quad\left|e_{4} \cap b\right|=2 .
$$

From previous observation it then directly follows that

$$
\left|e_{1} \cap b\right| \leqslant 1, \quad\left|e^{*} \cap b\right| \leqslant 1, \quad \text { and } \quad\left|e_{4} \cap a\right| \leqslant 1 .
$$

If $e^{*}$ intersects $e_{4}$ on two vertices, then $e^{*}$ intersects $e_{1}$ on at most one vertex and together with (A.1) we conclude $\left\{e_{1}, b, e^{*}\right\}$ is a 1-intersecting set, which is a contradiction. Thus, we have

$$
\left|e^{*} \cap e_{4}\right| \leqslant 1
$$

Next, let us look at the edge $e_{2}$. If $\left|e_{2} \cap a\right| \leqslant 1$ then since $\left|a \cap e_{4}\right| \leqslant 1$ by (A.1) and by the definition of the natural order of the edges $E(F)$ from the path $T_{6}^{3}$ we conclude that $\left\{e_{2}, a, e_{4}\right\}$ is a 1 -intersecting set, which is not possible. Thus, we know

$$
\left|e_{2} \cap a\right|=2 .
$$

Similarly, by (A.2) and the fact that $\left|e_{2} \cap e_{4}\right|=1$ we obtain

$$
\left|e_{2} \cap e^{*}\right|=2 .
$$

To finish the proof, it suffices to show that $H \backslash\left\{e_{1}, e_{3}\right\}$ does not contain a copy of $T_{6}^{3}$. Let $\tilde{F}$ be an arbitrary $T_{6}^{3}$-copy from $H \backslash\left\{e_{1}, e_{3}\right\}$. As there are only five edges in $H \backslash\left\{e_{1}, e_{3}\right\}$ either both $e_{2}$ and $b$ or both $e^{*}$ and $a$ are contained in $E(\tilde{F})$. We split the rest of the proof into these two cases:

Case 1: $\left\{e_{2}, b\right\} \subseteq E(\tilde{F})$

By (A.3) we know $\left|e_{2} \cap b\right| \leqslant 1$, but we show that actually $\left|e_{2} \cap b\right|=1$. It must be that $\left|e_{3} \cap b\right|=2$ as otherwise by (A.1) the set $\left\{e_{3}, b, e_{1}\right\}$ is 1 -intersecting. Since $\left|e_{3} \cap b\right|=2$ and $\left|e_{3} \cap e_{2}\right|=2$ we get $\left|e_{2} \cap b\right| \geqslant 1$ and thus $\left|e_{2} \cap b\right|=1$. By applying Lemma 30 to $\tilde{F}, e_{2}$ (as $a_{0}$ ) and $b$ (as $a_{1}$ ) we conclude that there exists an edge $x \in\left\{e_{4}, a, e^{*}\right\}$ such that $\left|x \cap e_{2}\right|=2$ and $|x \cap b|=2$. As $a \cap b=\emptyset,\left|e_{4} \cap e_{2}\right|=1$ and $\left|e^{*} \cap b\right| \leqslant 1$ we know that $x \notin\left\{e_{4}, a, e^{*}\right\}$, which is a contradiction.

Case 2: $\left\{e^{*}, a\right\} \subseteq E(\tilde{F})$

Since $\left|e^{*} \cap a\right|=2$ by applying Lemma 30 to $\tilde{F}, e^{*}$ (as $a_{0}$ ) and $a$ (as $a_{1}$ ) we conclude that there exist an edge $x \in\left\{b, e_{2}, e_{4}\right\}$ such that $|x \cap a|+\left|x \cap e^{*}\right|=3$. As $b \cap a=\emptyset$ it cannot be that $x=b$. Furthermore, using (A.3) and (A.4) we know that $\left|e_{2} \cap a\right|+\left|e_{2} \cap e^{*}\right|=4$ and thus $x \neq e_{2}$. Finally, using (A.1) and (A.2) we know $\left|e_{4} \cap a\right|+\left|e_{4} \cap e^{*}\right| \leqslant 2$ and thus $x \neq e_{4}$. This concludes the proof. 
Finally, by using lemma above we prove Lemma 29.

Proof of Lemma 29. If $H$ does not contain a 1-intersecting set of size three, then the lemma follows directly by Lemma 40. Let us assume that $H$ contains a 1intersecting set of size three $\{a, b, c\}$. Moreover, in order to arrive at contradiction we assume that for every 1-intersecting set $S \subseteq E(H)$ there exist a $T_{6}^{3}$-copy contained in $H \backslash S$. Consequently, as $|E(H) \backslash\{a, b, c\}|=4$, the hypergraph $H \backslash\{a$, $b, c\}$ must be isomorphic to $T_{6}^{3}$. Let $\left\{v_{1}, \ldots, v_{6}\right\}$ and $\left\{e_{1}, e_{2}, e_{3}, e_{4}\right\}$ be a natural order of vertices and edges of the $T_{6}^{3}$-copy spanned by $|E(H) \backslash\{a, b, c\}|$ such that $e_{i}=\left\{v_{i}, v_{i+1}, v_{i+2}\right\}$. We claim that either the edge $e_{1}$ or $e_{4}$ must be 1 -intersecting with at least two edges from $\{a, b, c\}$. If we assume otherwise, then there must be an edge in $\{a, b, c\}$, say $a$, such that both $e_{1}$ and $e_{4}$ intersect $a$ on two vertices. However, that is not possible as $e_{1} \cap e_{4}=\emptyset$. Without loss of generality, let us assume $\left\{e_{1}, a, b\right\}$ is a 1 -intersecting set. If $e_{1}$ is 1 -intersecting with all the edges from $\{a, b, c\}$, then $H \backslash\left\{e_{1}, a, b, c\right\}$ contains only three edges and cannot contain a $T_{6}^{3}$-copy, thus a contradiction. In the rest of the proof we consider the case when $\left|e_{1} \cap c\right|=2$.

Since $\left\{e_{1}, a, b\right\}$ is a 1 -intersecting set we know that $\left\{c, e_{2}, e_{3}, e_{4}\right\}$ induces a copy of $T_{6}^{3}$. Furthermore, the assumption $\left|e_{1} \cap c\right|=2$ implies $\left|c \cap e_{2}\right| \geqslant 1$. From $\left|e_{1} \cap e_{4}\right|=0$ we have $\left|c \cap e_{4}\right| \leqslant 1$. If $\left|c \cap e_{4}\right|=1$ and since $\left\{c, e_{2}, e_{3}, e_{4}\right\}$ induces a copy of $T_{6}^{3}$, it follows that $\left|c \cap e_{3}\right|=0$ and $\left|e_{2} \cap e_{4}\right|=2$, which is a contradiction to $\left|e_{2} \cap e_{4}\right|=1$. Therefore we obtain

$$
\left|c \cap e_{4}\right|=0 \quad \text { and } \quad\left|c \cap e_{2}\right|=2 .
$$

Next, as $e_{2}$ and $e_{4}$ are 1 -intersecting we know $H \backslash\left\{e_{2}, e_{4}\right\}$ contains a $T_{6}^{3}$-copy, denoted by $F$. To conclude the proof, we show that this is impossible. Let us now consider the following two cases:

Case 1: $\left\{e_{1}, e_{3}\right\} \nsubseteq E(F)$

Since at most one edge from $\left\{e_{1}, e_{3}\right\}$ is part of $F$ we conclude $\{a, b, c\} \subseteq E(F)$. This is a contradiction as by Observation 31 we know that $F$ cannot contain a 1-intersecting set of size three.

Case 2: $\left\{e_{1}, e_{3}\right\} \subseteq E(F)$

Using Lemma 30 we conclude that one of the edges from $E(F) \backslash\left\{e_{1}, e_{3}\right\}$ intersects both $e_{1}$ and $e_{3}$ on two vertices. Neither $a$ or $b$ can be such edge as they are 1-intersecting with $e_{1}$. On the other hand, we know $\left|c \cap e_{4}\right|=0$ which implies $\left|c \cap e_{3}\right| \leqslant 1$. Therefore no edge from set $E(F) \backslash\left\{e_{1}, e_{3}\right\}$ intersects both $e_{1}$ and $e_{3}$ on two vertices, which is a contradiction. 


\section{References}

[1] N. Alon and J. H. Spencer, The Probabilistic Method (John Wiley \& Sons, 2015).

[2] J. Balogh, R. Morris and W. Samotij, 'Independent sets in hypergraphs', J. Amer. Math. Soc. 28(3) (2015), 669-709.

[3] B. Bollobás, 'Threshold functions for small subgraphs', Math. Proc. Cambridge Philos. Soc. 90(2) (1981), 197-206.

[4] D. Conlon, D. Dellamonica Jr., S. La Fleur, V. Rödl and M. Schacht, 'A note on induced Ramsey numbers', Preprint (2016), arXiv:1601.01493, https://link.springer.com/chapter/10.1 007/978-3-319-44479-6_13.

[5] D. Conlon and W. T. Gowers, 'Combinatorial theorems in sparse random sets', Ann. of Math. (2) 184 (2016), 367-454.

[6] D. Conlon, W. T. Gowers, W. Samotij and M. Schacht, 'On the KŁR conjecture in random graphs', Israel J. Math. 203(1) (2014), 535-580.

[7] E. Friedgut, 'Hunting for sharp thresholds', Random Structures Algorithms 26(1-2) (2005), 37-51.

[8] E. Friedgut, H. Hàn, Y. Person and M. Schacht, 'A sharp threshold for van der Waerden's theorem in random subsets', Discrete Analysis 7 (2016), 20.

[9] E. Friedgut and M. Krivelevich, 'Sharp thresholds for certain Ramsey properties of random graphs', Random Structures Algorithms 17(1) (2000), 1-19.

[10] E. Friedgut, V. Rödl, A. Ruciński and P. Tetali, 'A sharp threshold for random graphs with a monochromatic triangle in every edge coloring', Mem. Amer. Math. Soc. 179(845) (2006), 66.

[11] E. Friedgut, V. Rödl and M. Schacht, 'Ramsey properties of random discrete structures', Random Structures Algorithms 37(4) (2010), 407-436.

[12] S. Janson, T. Łuczak and A. Ruciński, Random Graphs (Wiley, New York, 2000).

[13] Y. Kohayakawa and B. Kreuter, 'Threshold functions for asymmetric Ramsey properties involving cycles', Random Structures Algorithms 11(3) (1997), 245-276.

[14] Y. Kohayakawa, M. Schacht and R. Spöhel, 'Upper bounds on probability thresholds for asymmetric ramsey properties', Random Structures Algorithms 44(1) (2014), 1-28.

[15] T. Łuczak, A. Ruciński and B. Voigt, 'Ramsey properties of random graphs', J. Combin. Theory Ser. B 56(1) (1992), 55-68.

[16] M. Marciniszyn, J. Skokan, R. Spöhel and A. Steger, 'Asymmetric Ramsey properties of random graphs involving cliques', Random Structures Algorithms 34(4) (2009), 419-453.

[17] R. Nenadov, Y. Person, N. Škorić and A. Steger, 'An algorithmic framework for obtaining lower bounds for random Ramsey problems', J. Combin. Theory Ser. B 124 (2017), 1-38.

[18] R. Nenadov and A. Steger, 'A short proof of the random Ramsey theorem', Combin. Probab. Comput. 25(01) (2016), 130-144.

[19] R. Nenadov, A. Steger and M. Stojaković, 'On the threshold for the Maker-Breaker $H$-game', Random Structures Algorithms 49(3) (2016), 558-578.

[20] V. Rödl and A. Ruciński, 'Lower bounds on probability thresholds for Ramsey properties', in Combinatorics, Paul Erdös is eighty, 1, Vol. 1, Bolyai Society Mathematical Studies (János Bolyai Mathematical Society, 1993), 317-346.

[21] V. Rödl and A. Ruciński, 'Random graphs with monochromatic triangles in every edge coloring', Random Structures Algorithms 5(2) (1994), 253-270.

[22] V. Rödl and A. Ruciński, 'Threshold functions for Ramsey properties', J. Amer. Math. Soc. 8(4) (1995), 917-942. 
[23] V. Rödl and A. Ruciński, 'Ramsey properties of random hypergraphs', J. Combin. Theory Ser. A 81(1) (1998), 1-33.

[24] V. Rödl, A. Ruciński and M. Schacht, 'Ramsey properties of random $k$-partite, $k$-uniform hypergraphs', SIAM J. Discrete Math. 21(2) (2007), 442-460.

[25] V. Rödl, A. Ruciński and M. Schacht, 'An exponential-type upper bound for Folkman numbers', Combinatorica 37(4) (2017), 767-784.

[26] D. Saxton and A. Thomason, 'Hypergraph containers', Invent. Math. 201(3) (2015), 925-992.

[27] M. Schacht and F. Schulenburg, 'Sharp thresholds for Ramsey properties of strictly balanced nearly bipartite graphs', Random Structures Algorithms Preprint (2016), arXiv:1602.02501.

[28] H. Thomas, 'Aspects of games on random graphs', PhD Thesis, ETH Zurich, 2013. 